\title{
Density of states of chaotic Andreev billiards
}

\author{
Jack Kuipers, ${ }^{1, *}$ Thomas Engl, ${ }^{1, \dagger}$ Gregory Berkolaiko, ${ }^{2}$ Cyril Petitjean, ${ }^{1,3}$ Daniel Waltner, ${ }^{1}$ and Klaus Richter ${ }^{1}$ \\ ${ }^{1}$ Institut für Theoretische Physik, Universität Regensburg, D-93040 Regensburg, Germany \\ ${ }^{2}$ Department of Mathematics, Texas A\&M University, College Station, Texas 77843-3368, USA \\ ${ }^{3}$ SPSMS, UMR-E 9001, CEA-INAC/UJF-Grenoble 1, 17 rue des Martyrs, F-38054 Grenoble Cedex 9, France
}

(Received 20 April 2010; published 13 May 2011)

\begin{abstract}
Quantum cavities or dots have markedly different properties depending on whether their classical counterparts are chaotic or not. Connecting a superconductor to such a cavity leads to notable proximity effects, particularly the appearance, predicted by random matrix theory, of a hard gap in the excitation spectrum of quantum chaotic systems. Andreev billiards are interesting examples of such structures built with superconductors connected to a ballistic normal metal billiard since each time an electron hits the superconducting part it is retroreflected as a hole (and vice versa). Using a semiclassical framework for systems with chaotic dynamics, we show how this reflection, along with the interference due to subtle correlations between the classical paths of electrons and holes inside the system, is ultimately responsible for the gap formation. The treatment can be extended to include the effects of a symmetry-breaking magnetic field in the normal part of the billiard or an Andreev billiard connected to two phase-shifted superconductors. Therefore, we are able to see how these effects can remold and eventually suppress the gap. Furthermore, the semiclassical framework is able to cover the effect of a finite Ehrenfest time, which also causes the gap to shrink. However, for intermediate values this leads to the appearance of a second hard gap - a clear signature of the Ehrenfest time.
\end{abstract}

DOI: 10.1103/PhysRevB.83.195316

PACS number(s): 74.40.-n, 03.65.Sq, 05.45.Mt, 74.45.+c

\section{INTRODUCTION}

The physics of normal metals $(\mathrm{N})$ in contact with superconductors (S) has been studied extensively for almost 50 years, and in the past two decades there has been somewhat of a resurgence of interest in this field. This has mainly been sparked by the realization of experiments that can directly probe the region close to the normal-superconducting (NS) interface at temperatures far below the transition temperature of the superconductor. Such experiments have been possible thanks to microlithographic techniques that permit the building of heterostructures on a mesoscopic scale combined with transport measurements in the sub-Kelvin regime. Such hybrid structures exhibit various new phenomena, mainly due to the fact that physical properties of both the superconductor and the mesoscopic normal metal are strongly influenced by quantum coherence effects.

The simplest physical picture of this system is that the superconductor tends to export some of its anomalous properties across the interface over a temperature-dependent length scale that can be of the order of a micrometer at low temperatures. This is the so-called proximity effect, which has been the focus on numerous surveys, both experimental ${ }^{1-9}$ and theoretical. ${ }^{10-13}$

The key concept to understand this effect ${ }^{14-16}$ is Andreev reflection. During this process, when an electron from the vicinity of the Fermi energy $\left(E_{F}\right)$ surface of the normal conductor hits the superconductor, the bulk energy gap $\Delta$ of the superconductor prevents the negative charge from entering, unless a Cooper pair is formed in the superconductor. Since a Cooper pair is composed of two electrons, an extra electron has to be taken from the Fermi sea, thus creating a hole in the conduction band of the normal metal. Physically and classically speaking, an Andreev reflection, therefore, corresponds to a retroflection of the particle, where Andreev reflected electrons (or holes) retrace their trajectories as holes (or electrons). The effect of Andreev reflection on the transport properties of open NS structures is an interesting and fruitful area (see Refs. 17 and 18 and references therein for example), though in this paper we focus instead on closed structures. Naturally, this choice has the consequence of leaving aside some exciting recent results such as, for example, the statistical properties of the conductance, ${ }^{19}$ the magnetoconductance in Andreev quantum dots, ${ }^{20}$ resonant tunneling, ${ }^{21}$ and the thermoelectric effect ${ }^{22,23}$ in Andreev interferometers.

In closed systems, one of the most noticeable manifestations of the proximity effect is the suppression of the density of states (DOS) of the normal metal just above the Fermi energy. Although most of the experimental investigations have been carried out on disordered systems, ${ }^{1,3,5,6,8}$ with recent technical advances, interest has moved to structures with clean ballistic dynamics. ${ }^{2,4,7,9,24,25}$ This shift gives access to the experimental investigation of the so-called Andreev billiard. While this term was originally coined ${ }^{26}$ for an impurity-free normal conducting region entirely confined by a superconducting boundary, it also refers to a ballistic normal area (i.e., a quantum dot) with a boundary that is only partly connected to a superconductor. The considerable theoretical attention raised by such a hybrid structure in the past decade is related to the interesting peculiarity that by looking at the DOS of an Andreev billiard, we can determine the nature of the underlying dynamics of its classical counterpart. ${ }^{27}$ Indeed, while the DOS vanishes with a power law in energy for the integrable case, the spectrum of a chaotic billiard is expected to exhibit a true gap above $E_{F} .{ }^{27}$ The width of this hard gap, also called the minigap, ${ }^{13}$ has been calculated as a purely quantum effect by using random matrix theory (RMT) and its value scales with the Thouless energy, $E_{T}=\hbar / 2 \tau_{\mathrm{d}}$, where $\tau_{\mathrm{d}}$ is the average (classical) dwell time a particle stays in the billiard between successive Andreev reflections. ${ }^{27}$ 
Since the existence of this gap is expected to be related to the chaotic nature of the electronic motion, many attempts have been undertaken to explain this result in semiclassical terms ${ }^{28-34}$ however this appeared to be rather complicated. Indeed, a traditional semiclassical treatment based on the so-called Bohr-Sommerfeld (BS) approximation yields only an exponential suppression of the DOS. ${ }^{28-30}$ This apparent contradiction of this prediction with the RMT one was resolved quite early by Lodder and Nazarov, ${ }^{28}$ who pointed out the existence of two different regimes. The characteristic time scale that governs the crossover between the two regimes is the Ehrenfest time $\tau_{\mathrm{E}} \sim|\ln \hbar|$, which is the time scale that separates the evolution of wave packets following essentially the classical dynamics from longer time scales dominated by wave interference. In particular, it is the ratio $\tau=\tau_{\mathrm{E}} / \tau_{\mathrm{d}}$ that has to be considered.

In the universal regime, $\tau=0$, chaos sets in sufficiently rapidly and RMT is valid, leading to the appearance of the aforementioned Thouless gap. ${ }^{27}$ Although the Thouless energy $E_{T}$ is related to a purely classical quantity, namely the average dwell time, we stress that the appearance of the minigap is a quantum-mechanical effect, and consequently the gap closes if a symmetry-breaking magnetic field is applied. ${ }^{35}$ Similarly, if two superconductors are attached to the Andreev billiard, the size of the gap will depend on the relative phase between the two superconductors, with the gap vanishing for a $\pi$ junction. ${ }^{35}$

The deep classical limit is characterized by $\tau \rightarrow \infty$, and in this regime the suppression of the DOS is exponential and well described by the BS approximation. The more interesting crossover regime of finite Ehrenfest time, and the conjectured Ehrenfest time gap dependence of Ref. 28, have been investigated by various means. ${ }^{12,21,36-40}$ Due to the logarithmic nature of $\tau_{\mathrm{E}}$, investigating numerically the limit of large Ehrenfest time is rather difficult, but a clear signature of the gap's Ehrenfest time dependence has been obtained $^{41-43}$ for $\tau<1$. From an analytical point of view, RMT is inapplicable in the finite $\tau_{\mathrm{E}}$ regime, ${ }^{12}$ therefore new methods such as a stochastic method ${ }^{38}$ using smooth disorder and sophisticated perturbation methods that include diffraction effects ${ }^{36}$ have been used to tackle this problem. On the other hand, a purely phenomenological model, effective RMT, has been developed ${ }^{37,44}$ and predicts a gap size scaling with the Ehrenfest energy $E_{\mathrm{E}}=\hbar / 2 \tau_{\mathrm{E}}$. Recently, Micklitz and Altland, ${ }^{40}$ based on a refinement of the quasiclassical approach and the Eilenberger equation, succeeded to show the existence of a gap of width $\pi E_{\mathrm{E}} \propto 1 / \tau$ in the limit of large $\tau \gg 1$.

Consequently, a complete picture of all the available regimes was still missing until recently, when we treated the DOS semiclassically ${ }^{45}$ following the scattering approach. ${ }^{46}$ Starting for $\tau=0$ and going beyond the diagonal approximation, we used an energy-dependent generalization of the work $^{47}$ on the moments of the transmission eigenvalues. The calculation is based on the evaluation of correlation functions also appearing in the moments of the Wigner delay times. ${ }^{48}$ More importantly, the effect of finite Ehrenfest time could be incorporated in this framework ${ }^{49}$ leading to a microscopic confirmation of the $\tau_{\mathrm{E}}$ dependence of the gap predicted by effective RMT. Interestingly the transition between $\tau=0$ and $\tau=\infty$ is not smooth, and a second gap at $\pi E_{\mathrm{E}}$ was observed for intermediate $\tau$, providing us with certainly the most clear-cut signature of Ehrenfest time effects.

In this paper, we extend and detail the results obtained in Ref. 45. First we discuss Andreev billiards and their treatment using RMT and semiclassical techniques. For the DOS in the universal regime $(\tau=0)$, we first delve into the work of Refs. 47 and 48 before using it to obtain the generating function of the correlation functions that are employed to derive the DOS. This is done both in the absence and in the presence of a time-reversal symmetry-breaking magnetic field, and we also look at the case in which the bulk superconducting gap and the excitation energy of the particle are comparable.

We then treat Andreev billiards connected to two superconducting contacts with a phase difference $\phi$. The gap is shown to shrink with increasing phase difference due to the accumulation of a phase along the trajectories that connect the two superconductors. Finally, the Ehrenfest regime will be discussed, especially the appearance of a second intermediate gap for a certain range of $\tau$. We will also show that this intermediate gap is very sensitive to the phase difference between the superconductors.

\section{ANDREEV BILLIARDS}

Since the treatment of Andreev billiards was recently reviewed in Ref. 13, we just recall some useful details here. In particular, the chaotic Andreev billiard that we consider is treated within the scattering approach ${ }^{46}$ where the NS interface is modeled with the help of a fictitious ideal lead. This lead permits the contact between the normal metal cavity (with chaotic classical dynamics) and the semi-infinite superconductor as depicted in Fig. 1(a).

Using the continuity of the superconducting and normal wave function, we can construct the scattering matrix of the whole system. Denoting the excitation energy of the electron above the Fermi energy $E_{F}$ by $E$ and assuming that the lead supports $N$ channels (transverse modes at the Fermi energy), the scattering matrix of the whole normal region can be written in a joint electron-hole basis and reads

$$
S_{\mathrm{N}}(E)=\left(\begin{array}{cc}
S(E) & 0 \\
0 & S^{*}(-E)
\end{array}\right),
$$
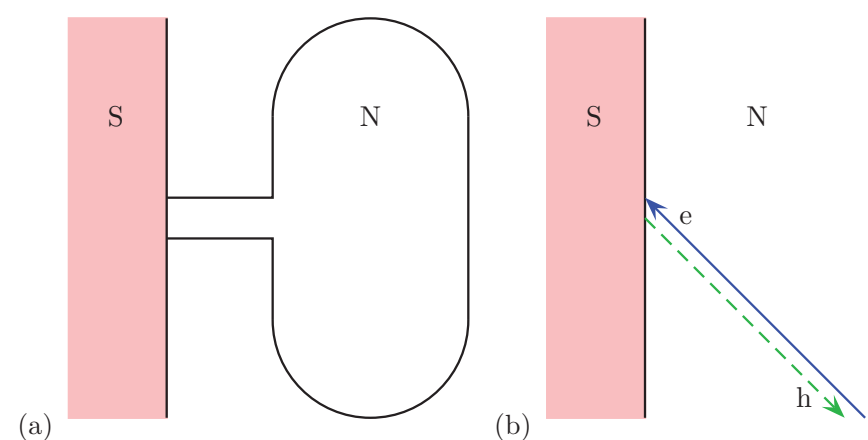

FIG. 1. (Color online) (a) The Andreev billiard consists of a chaotic normal metal $(\mathrm{N})$ cavity attached to a superconductor $(\mathrm{S})$ via a lead. (b) At the NS interface between the normal metal and the superconductor, electrons are retroreflected as holes. 
where $S(E)$ is the unitary $N \times N$ scattering matrix of the electrons [and its complex conjugate $S^{*}(-E)$ that of the holes]. As the electrons and holes remain uncoupled in the normal region, the off-diagonal blocks are zero. Instead, electrons and holes couple at the NS interface through Andreev reflection, ${ }^{15}$ where electrons are retroreflected as holes and vice versa, as in Fig. 1(b). For energies $E$ smaller than the bulk superconductor gap $\Delta$, there is no propagation into the superconductor, and if we additionally assume $\Delta \ll E_{F}$, we can encode the Andreev reflection in the matrix,

$$
\begin{gathered}
S_{\mathrm{A}}(E)=\alpha(E)\left(\begin{array}{ll}
0 & 1 \\
1 & 0
\end{array}\right), \\
\alpha(E)=\mathrm{e}^{-\mathrm{i} \arccos \left(\frac{E}{\Delta}\right)}=\frac{E}{\Delta}-\mathrm{i} \sqrt{1-\frac{E^{2}}{\Delta^{2}}} .
\end{gathered}
$$

The retroreflection (of electrons as holes with the same channel index) is accompanied by the phase shift $\arccos (E / \Delta)$. In the limit of perfect Andreev reflection $(E=0)$, this phase shift reduces to $\pi / 2$.

Below $\Delta$, the Andreev billiard has a discrete excitation spectrum at energies where $\operatorname{det}\left[1-S_{\mathrm{A}}(E) S_{\mathrm{N}}(E)\right]=0$, which can be simplified ${ }^{46}$ to

$$
\operatorname{det}\left[1-\alpha^{2}(E) S(E) S^{*}(-E)\right]=0 .
$$

Finding the roots of this equation yields the typical density of states of chaotic Andreev billiards. In the next two sections, we review the two main analytical frameworks that can be used to tackle this problem.

\section{A. Random matrix theory}

One powerful treatment uses random matrix theory. Such an approach was initially considered in Refs. 27 and 35, where the actual setup treated is depicted in Fig. 2(a). It consists of a normal metal $(\mathrm{N})$ connected to two superconductors $\left(\mathrm{S}_{1}, \mathrm{~S}_{2}\right)$ by narrow leads carrying $N_{1}$ and $N_{2}$ channels. The superconductors' order parameters are considered to have phases $\pm \phi / 2$, with a total phase difference $\phi$. Moreover, a perpendicular magnetic field $B$ was applied to the normal part. We note that although this figure [and Fig. 1(a)] have spatial symmetry, the treatment is actually for the case without such symmetry.

As above, the limit $\Delta \ll E_{F}$ was taken so that normal reflection at the NS interface can be neglected and the

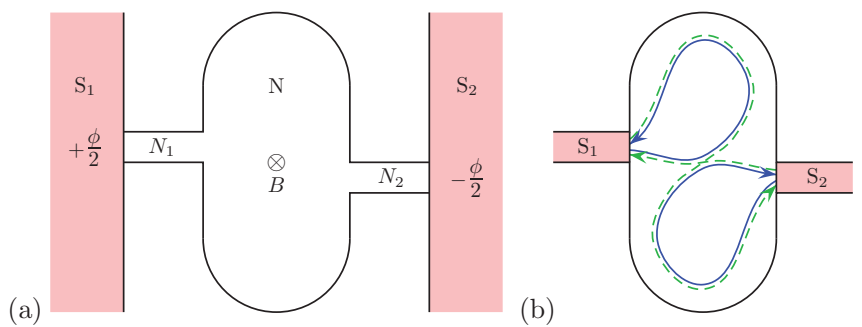

FIG. 2. (Color online) (a) An Andreev billiard connected to two superconductors $\left(\mathrm{S}_{1}, \mathrm{~S}_{2}\right)$ at phases $\pm \phi / 2$ via leads carrying $N_{1}$ and $N_{2}$ channels, all threaded by a perpendicular magnetic field $B$. (b) The semiclassical treatment involves classical trajectories retroreflected at the superconductors an arbitrary number of times. symmetric case in which both leads contain the same number, $N / 2$, of channels was considered. ${ }^{27,35}$ Finally, it was also assumed that $\alpha \approx-\mathrm{i}$, valid in the limit $E, E_{T} \ll \Delta \ll E_{F}$. For such a setup, the determinantal equation (4) becomes

$$
\operatorname{det}\left[1+S(E) \mathrm{e}^{\mathrm{i} \tilde{\phi}} S^{*}(-E) \mathrm{e}^{-\mathrm{i} \tilde{\phi}}\right]=0,
$$

where $\tilde{\phi}$ is a diagonal matrix whose first $N / 2$ elements are $\phi / 2$ and the remaining $N / 2$ elements $-\phi / 2$. We note that though we stick to the case of perfect coupling here, the effect of tunnel barriers was also included in Ref. 27.

The first step is to rewrite the scattering problem in terms of a low-energy effective Hamiltonian $\mathcal{H}$,

$$
\mathcal{H}=\left(\begin{array}{cc}
\hat{H} & \pi X X^{T} \\
-\pi X X^{T} & -\hat{H}^{*}
\end{array}\right),
$$

where $\hat{H}$ is the $M \times M$ Hamiltonian of the isolated billiard and $X$ an $M \times N$ coupling matrix. Eventually, the limit $M \rightarrow$ $\infty$ is taken, and to mimic a chaotic system, the matrix $\hat{H}$ is replaced by a random matrix following the Pandey-Mehta distribution, ${ }^{17}$

$P(H) \propto \exp \left\{-\frac{N^{2}\left(1+a^{2}\right)}{64 M E_{T}^{2}} \sum_{i, j=1}^{M}\left[\left(\operatorname{Re} \hat{H}_{i j}\right)^{2}+a^{-2}\left(\operatorname{Im} \hat{H}_{i j}\right)^{2}\right]\right\}$.

The parameter $a$ measures the strength of the time-reversal symmetry breaking, so we can investigate the crossover from the ensemble with time-reversal symmetry, the Gaussian orthogonal ensemble (GOE), to that without, the Gaussian unitary ensemble (GUE). It is related to the magnetic flux $\Phi$ through the two-dimensional billiard of area $A$ and with Fermi velocity $v_{F}$ by

$$
M a^{2}=c\left(\frac{e \Phi}{h}\right)^{2} \hbar v_{F} \frac{N}{2 \pi E_{T} \sqrt{A}} .
$$

Here $c$ is a numerical constant of order unity depending only on the shape of the billiard. The critical flux is then defined via

$$
M a^{2}=\frac{N}{8}\left(\frac{\Phi}{\Phi_{\mathrm{c}}}\right)^{2} \Leftrightarrow \Phi_{\mathrm{c}} \approx \frac{h}{e}\left(\frac{2 \pi E_{T}}{\hbar v_{F}}\right)^{\frac{1}{2}} A^{\frac{1}{4}} .
$$

The density of states, divided for convenience by twice the mean density of states of the isolated billiard, can be written as

$$
d(\epsilon)=-\operatorname{Im} W(\epsilon),
$$

where $W(\epsilon)$ is the trace of a block of the Green function of the effective Hamiltonian of the scattering system, and for simplicity here we express the energy in units of the Thouless energy $\epsilon=E / E_{T}$. This is averaged by integrating over (7) using diagrammatic methods, ${ }^{50}$ which to leading order in inverse channel number $1 / N$ leads to the expression ${ }^{35}$

$$
W(\epsilon)=\left(\frac{b}{2} W(\epsilon)-\frac{\epsilon}{2}\right)\left(1+W^{2}(\epsilon)+\frac{\sqrt{1+W^{2}(\epsilon)}}{\beta}\right),
$$

where $\beta=\cos (\phi / 2)$ and $b=\left(\Phi / \Phi_{\mathrm{c}}\right)^{2}$ with the critical magnetic flux $\Phi_{\mathrm{c}}$ for which the gap in the density of states closes (at $\phi=0$ ). Eq. (11) may also be rewritten as a sixth-order 
polynomial, and when substituting into (10), we should take the solution that tends to 1 for large energies. In particular, when there is no phase difference between the two leads ( $\phi=0$, or equivalently when we consider a single lead carrying $N$ channels) and no magnetic field in the cavity $\left(\Phi / \Phi_{c}=0\right)$, the density of states is given by a solution of the cubic equation,

$$
\epsilon^{2} W^{3}(\epsilon)+4 \epsilon W^{2}(\epsilon)+\left(4+\epsilon^{2}\right) W(\epsilon)+4 \epsilon=0 .
$$

\section{B. Semiclassical approach}

The second approach, and that which we pursue and detail in this paper, is to use the semiclassical approximation to the scattering matrix, which involves the classical trajectories that enter and leave the cavity. ${ }^{51}$ Using the general expression between the density of states and the scattering matrix, ${ }^{52}$ the density of states of an Andreev billiard reads ${ }^{30,46,53}$

$$
\tilde{d}(E)=\bar{d}-\frac{1}{\pi} \operatorname{Im} \frac{\partial}{\partial E} \ln \operatorname{det}\left[1-S_{\mathrm{A}}(E) S_{\mathrm{N}}(E)\right],
$$

where $\bar{d}=N / 2 \pi E_{T}$ is twice the mean density of states of the isolated billiard (around the Fermi energy). Eq. (13) should be understood as an averaged quantity over a small range of the Fermi energy or slight variations of the billiard, and for convergence reasons a small imaginary part is included in the energy $E$. In the limit of perfect Andreev reflection, $\alpha(E) \approx-\mathrm{i}$, see (3), and (13) reduces to

$$
\tilde{d}(E)=\bar{d}+\frac{1}{\pi} \operatorname{Im} \frac{\partial}{\partial E} \operatorname{Tr} \sum_{m=1}^{\infty} \frac{1}{m}\left(\begin{array}{cc}
0 & \mathrm{i} S^{*}(-E) \\
\mathrm{i} S(E) & 0
\end{array}\right)^{m} .
$$

Obviously only even terms in the sum have a nonzero trace, and setting $n=2 m$, dividing through by $\bar{d}$, and expressing the energy in units of the Thouless energy $\epsilon=E / E_{T}$, this simplifies to $^{30}$

$$
d(\epsilon)=1+2 \operatorname{Im} \sum_{n=1}^{\infty} \frac{(-1)^{n}}{n} \frac{\partial C(\epsilon, n)}{\partial \epsilon} .
$$

Eq. (15) involves the correlation functions of $n$ scattering matrices

$$
C(\epsilon, n)=\frac{1}{N} \operatorname{Tr}\left[S^{*}\left(-\frac{\epsilon \hbar}{2 \tau_{\mathrm{d}}}\right) S\left(\frac{\epsilon \hbar}{2 \tau_{\mathrm{d}}}\right)\right]^{n},
$$

where we recall that the energy is measured relative to the Fermi energy and that $E_{T}=\hbar / 2 \tau_{\mathrm{d}}$ involves the average dwell time $\tau_{\mathrm{d}}$. For chaotic systems, ${ }^{54}$ the dwell time can be expressed as $\tau_{\mathrm{d}}=T_{\mathrm{H}} / N$ in terms of the Heisenberg time $T_{\mathrm{H}}$ conjugate to the mean level spacing $(2 / \bar{d})$.

At this point, it is important to observe that nonzero values of $\epsilon$ are necessary for the convergence of the expansion of the logarithm in (13) that led to (15). On the other hand, we are particularly interested in small values of $\epsilon$, which put (15) on the edge of the radius of convergence, where it is highly oscillatory. The oscillatory behavior and a slow decay in $n$ are a direct consequence of the unitarity of the scattering matrix at $\epsilon=0$ (in fact, later it can also be shown that $\left.\left.\frac{\partial C(\epsilon, n)}{\partial \epsilon}\right|_{\epsilon=0}=\mathrm{i} n\right)$. Thus a truncation of (15) will differ markedly from the predicted RMT gap, which was the root of the difficulty of capturing the gap by previous semiclassical treatments..$^{30,33,34}$ In the present work, we succeed in evaluating the entire sum and hence obtain results that are uniformly valid for all values of $\epsilon$.

Calculating the density of states is then reduced to the seemingly more complicated task of evaluating correlation functions semiclassically for all $n$. Luckily the treatment of such functions has advanced rapidly in the past few years $^{47,48,55-57}$ and we build on that solid basis. We also note that determining $C(\epsilon, n)$ is a more general task than calculating the density of states. Since the Andreev reflection has already been encoded in the formalism before (15), the treatment of the $C(\epsilon, n)$ no longer depends on the presence or absence of the superconducting material, but solely on the properties of the chaotic dynamics inside the normal metal billiard.

In the semiclassical approximation, the elements of the scattering matrix are given by ${ }^{51}$

$$
S_{o i}(E) \approx \frac{1}{\sqrt{T_{\mathrm{H}}}} \sum_{\zeta(i \rightarrow o)} A_{\zeta} \mathrm{e}^{\mathrm{i} S_{\zeta}(E) / \hbar}
$$

where the sum runs over all classical trajectories $\zeta$ starting in channel $i$ and ending in channel $o . S_{\zeta}(E)$ is the classical action of the trajectory $\zeta$ at energy $E$ above the Fermi energy, and the amplitude $A_{\zeta}$ contains the stability of the trajectory as well as the Maslov phases. ${ }^{58}$ After we substitute (17) into (16) and expand the action around the Fermi energy up to first order in $\epsilon$ using $\partial S_{\zeta} / \partial E=T_{\zeta}$, where $T_{\zeta}$ is the duration of the trajectory $\zeta$, the correlation functions are given semiclassically by a sum over $2 n$ trajectories,

$$
\begin{aligned}
C(\epsilon, n) \approx & \frac{1}{N T_{\mathrm{H}}^{n}} \prod_{j=1}^{n} \sum_{i_{j}, o_{j}} \sum_{\substack{\zeta_{j}\left(i_{j} \rightarrow o_{j}\right) \\
\zeta_{j}^{\prime}\left(o_{j} \rightarrow i_{j+1}\right)}} A_{\zeta_{j}} A_{\zeta_{j}^{\prime}}^{*} \mathrm{e}^{\mathrm{i}\left(S_{\zeta_{j}}-S_{\zeta_{j}^{\prime}}\right) / \hbar} \\
& \times \mathrm{e}^{\mathrm{i} \epsilon\left(T_{\zeta_{j}}+T_{\zeta_{j}^{\prime}}\right) /\left(2 \tau_{\mathrm{d}}\right)} .
\end{aligned}
$$

The final trace in (16) means that we identify $i_{n+1}=i_{1}$, and as the electron trajectories $\zeta_{j}$ start at channel $i_{j}$ and end in channel $o_{j}$ while the primed hole trajectories $\zeta_{j}^{\prime}$ go backward starting in channel $o_{j}$ and ending in channel $i_{j+1}$, the trajectories fulfill a complete cycle, as in Figs. 3(a) 4(a), 4(d), and $4(\mathrm{~g})$. The channels $i_{1}, \ldots, i_{n}$ will be referred to as incoming channels, while $o_{1}, \ldots, o_{n}$ will be called outgoing channels. This refers to the direction of the electron trajectories at the channels and not necessarily to which lead the channel finds itself in (when we have two leads as in Fig. 2).

The actions in (18) are taken at the Fermi energy, and the resulting phase is given by the difference of the sum of the actions of the unprimed trajectories and the sum of the actions of the primed ones. In the semiclassical limit of $\hbar \rightarrow 0$ (cf. the RMT limit of $M \rightarrow \infty$ ), this phase oscillates widely, leading to cancellations when the averaging is applied, unless this total action difference is of the order of $\hbar$. The semiclassical treatment then involves finding sets of classical trajectories that can have such a small action difference and hence contribute consistently in the limit $\hbar \rightarrow 0$. 
(a)

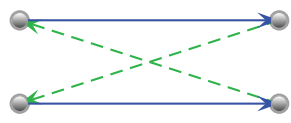

(b)

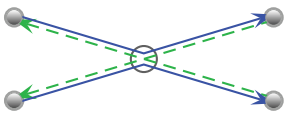

(c)

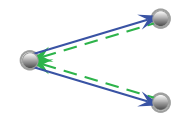

(d)

FIG. 3. (Color online) (a) The original trajectory structure of the correlation function $C(\epsilon, 2)$, where the incoming channels are drawn on the left, outgoing channels on the right, electrons as solid (blue), and holes as dashed (green) lines. (b) By collapsing the electron trajectories directly onto the hole trajectories, we create a structure where the trajectories only differ in a small region called an encounter. Placed inside the Andreev billiard, this diagram corresponds to Fig. 2(b). The encounter can be slid into the incoming channels on the left (c) or the outgoing channels on the right $(\mathrm{d})$ to create diagonal-type pairs.

\section{SEMICLASSICAL DIAGRAMS}

As an example, we show the original trajectory structure for $n=2$ in Fig. 3(a), where for convenience we draw the incoming channels on the left and the outgoing channels on the right so that electrons travel to the right and holes to the left (cf. the shot noise in Refs. 59-61). Of course the channels are really in the lead [Fig. 1(a)] or either lead (Fig. 2) and the trajectory stretches involve many bounces at the normal boundary of the cavity. We draw such topological sketches as the semiclassical methods were first developed for transport, ${ }^{47,55,57}$ where typically we have $S^{\dagger}$ (complex conjugate transpose) instead of $S^{*}$ (complex conjugate) in (16), restricted to the transmission subblocks, so that all the trajectories would travel to the right in our sketches. Without the magnetic field, the billiard has time-reversal symmetry and $S$ is symmetric, but this difference plays a role when we turn the magnetic field on later. An even more important difference is that in our problem, any channel can be in any lead.
To obtain a small action difference, and a possible contribution in the semiclassical limit, the trajectories must be almost identical. This can be achieved, for example, by collapsing the electron trajectories directly onto the hole trajectories as in Fig. 3b. Inside the open circle, the holes still "cross" while the electrons "avoid crossing," but by bringing the electron trajectories arbitrarily close together, the set of trajectories can have an arbitrary small action difference. More accurately, the existence of partner trajectories follows from the hyperbolicity of the phase-space dynamics. Namely, given two electron trajectories that come close (have an encounter) in the phase space, one uses the local stable and unstable manifolds ${ }^{62-64}$ to find the coordinates through which hole trajectories arrive along one electron trajectory and leave along the other, exactly as in Fig. 3(b) [and Fig. 2(b)]. These are the partner trajectories we pick for $\zeta_{1}^{\prime}$ and $\zeta_{2}^{\prime}$ when we evaluate $C(\epsilon, 2)$ from $(18)$ in the semiclassical approximation. As the encounter involves two electron trajectories, it is called a 2-encounter. An encounter can happen anywhere along the length of a trajectory. In

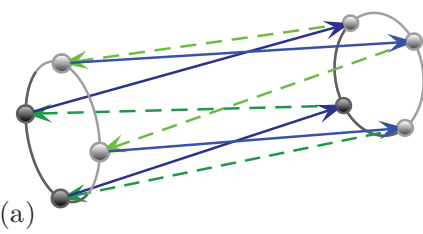

(a)

(b)

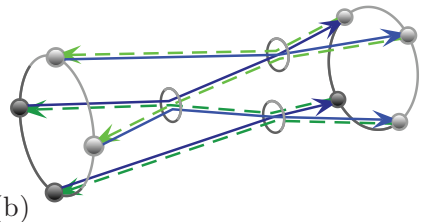

(c)
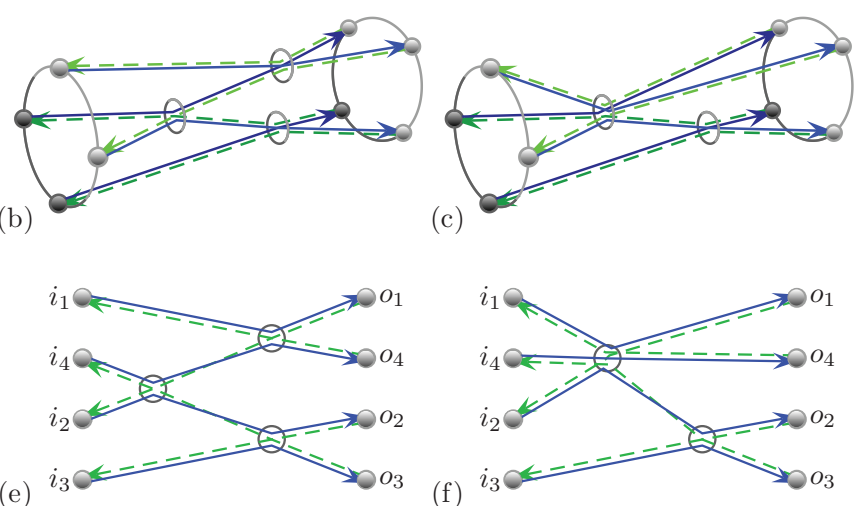

(f)
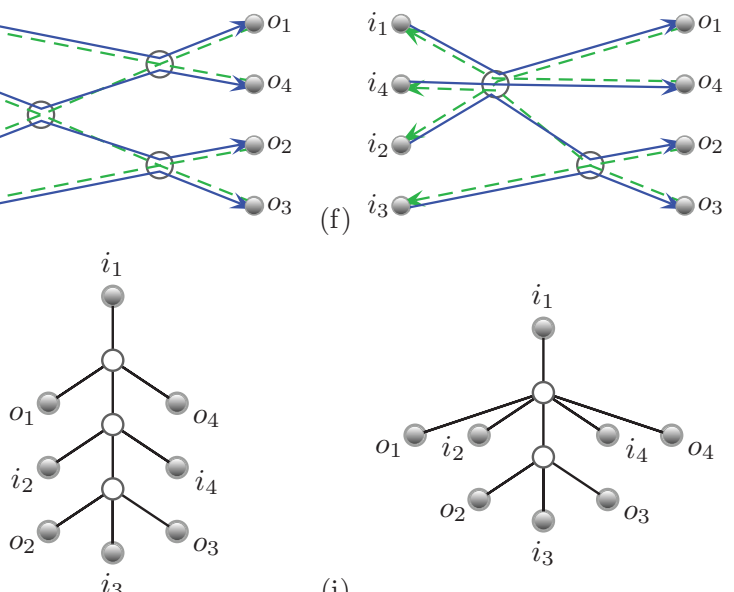

(h)

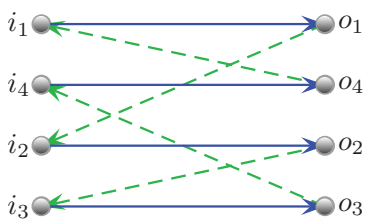

(g)

)

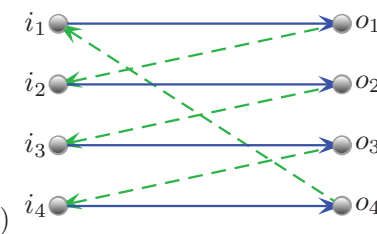

FIG. 4. (Color online) (a) The original trajectory structure of the correlation function $C(\epsilon, 4)$ where the incoming channels are drawn on the left, outgoing channels on the right, electrons as solid (blue), and holes as dashed (green) lines. (d,g) Equivalent 2D projections of the starting structure as the order is determined by moving along the closed cycle of electron and hole trajectories. (b) By pinching together the electron trajectories (pairwise here), we can create a structure that only differs in three small regions (encounters) and which can have a small action difference. (e) Projection of (b) also created by collapsing the electron trajectories in (g) directly onto the hole trajectories. (c,f) Sliding two of the encounters from (b) together (or originally pinching three electron trajectories together) creates these diagrams. (h,i) Resulting rooted plane tree diagrams of $(e, f)$ or $(b, c)$ defining the top left as the first incoming channel [i.e., the channel ordering as depicted in (e,f)]. 


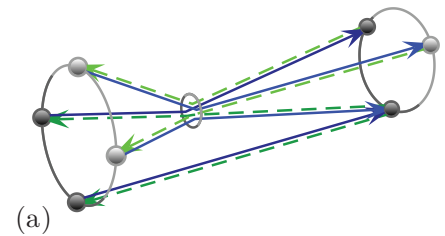

(b)

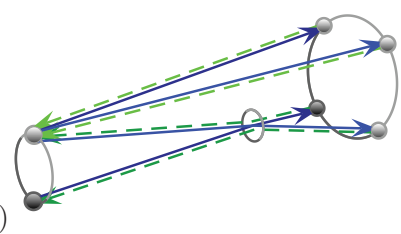

e)

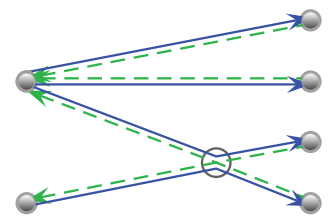

(c)

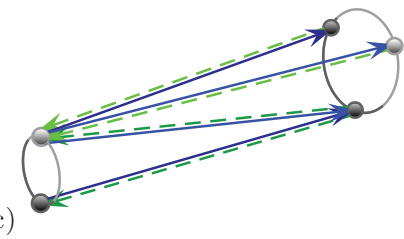

(f)

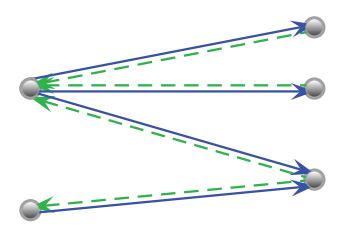

FIG. 5. (Color online) Further possibilities arise from moving encounters into the lead(s). Starting from Fig. 4(c), we can slide the 2-encounter into the outgoing channels on the right (called " $o$-touching," see the text) to arrive at (a,d) or the 3-encounter into the incoming channels on the left (called " $i$-touching") to obtain (b,e). Moving both encounters leads to (c,f), but moving both to the same side means first combining the 3- and 2-encounter in Fig. 4(c) into a 4-encounter, and it is treated as such.

particular, it can happen at the very beginning or the very end of a trajectory, in which case it is actually happening next to the lead; see Figs. 3(c) and 3(d). This situation is important as it will give an additional contribution to that of an encounter happening in the body of the billiard. We will refer to this situation as an "encounter entering the lead." We note that if an encounter enters the lead, the corresponding channels must coincide and we have diagonal-type pairs (i.e., the trajectories are coupled exactly pairwise), though it is worth bearing in mind that there is still a partial encounter happening near the lead, as shown by the Ehrenfest time treatment. ${ }^{60,65}$

To give a more representative example, consider the structure of trajectories for $n=4$. For visualization purposes, in Fig. 4(a) the original trajectories are arranged around a cylinder in the form of a cat's cradle. The incoming and outgoing channels are ordered around the circles at either end, although they could physically be anywhere. Projecting the structure into two dimensions (2D), we can draw it in several equivalent ways, for example as in Fig. 4(d) or 4(g), and we must take care not to overcount such equivalent representations. We note that the ordering of the channels is uniquely defined by the closed cycle that the trajectories form. To create a small action difference, we can imagine pinching together the electron (and hole) strings in Fig. 4(a). One possibility is to pinch two together in three places (making three 2-encounters), as in Fig. 4(b). A possible representation in 2D is shown in Fig. 4(e), which can also be created by collapsing the electron trajectories directly onto the hole trajectories in Fig. 4(g). Note that the collapse of the diagram in Fig. 4(d) leads to a different structure with three 2-encounters. However, in general it is not true that the different projections of the arrangement in Fig. 4(a) are in a one-to-one correspondence with all possible diagrams.

From Figs. 4(b) and 4(e), we can create another possibility by sliding two of the 2-encounters together to make a 3 -encounter [or alternatively, we could start by pinching three trajectories together in Fig. 4(a) as well as an additional pair] as in Fig. 4(c) and 4(f). Finally, we could combine both to a single 4-encounter. Along with the possibilities where all the encounters are inside the system, we can progressively slide encounters into the leads, as we did for the $n=2$ case in Fig. 3, creating, among others, the diagrams in Fig. 5.

Finally, we mention that so far we were listing only "minimal" diagrams. One can add more encounters to the above diagrams, but we will see later that such arrangements contribute at a higher order in the inverse number of channels and are therefore subdominant. The complete expansion in this small parameter is available only for small values of $n$; see Refs. 56, 59, and 57.

\section{A. Tree recursions}

To summarize the previous paragraph, the key task now is to generate all possible minimal encounter arrangements (see, for example, Ref. 48 for the complete list of those with $n=3$ ). This is a question that was answered in Ref. 47, where the moments of the transmission amplitudes were considered. The pivotal step was to redraw the diagrams as rooted plane trees and to show that there is a one-to-one relation between them (for the diagrams that contribute at leading order in inverse channel number). To redraw a diagram as a tree, we start with a particular incoming channel $i_{1}$ as the root (hence rooted trees) and place the remaining channels in order around an anticlockwise loop (hence plane). Moving along the trajectory $\zeta_{1}$, we draw each stretch as a link and each encounter as a node (open circle) until we reach $o_{1}$. Then we move along $\zeta_{1}^{\prime}$ back to its first encounter and continue along any new encounters to $i_{2}$, and so on. For example, the tree corresponding to Figs. 4(b) and 4(e) is drawn in Fig. 4(h), and that corresponding to Figs. 4(c) and 4(f) is in Fig. 4(i). Note that marking the root only serves to eliminate overcounting, and the final results do not depend on the particular choice of the root.

A particularly important property of the trees is their amenability to recursive counting. The recursions behind our treatment of Andreev billiards were derived in Ref. 47, and we recall the main details here. First we can describe the encounters in a particular tree by a vector $\boldsymbol{v}$ whose elements $v_{l}$ count the number of $l$-encounters in the tree (or diagram); this is often written as $2^{v_{2}} 3^{v_{3}} \ldots$. An $l$-encounter is a vertex in the tree of degree $2 l$ (i.e., connected to $2 l$ links). The 

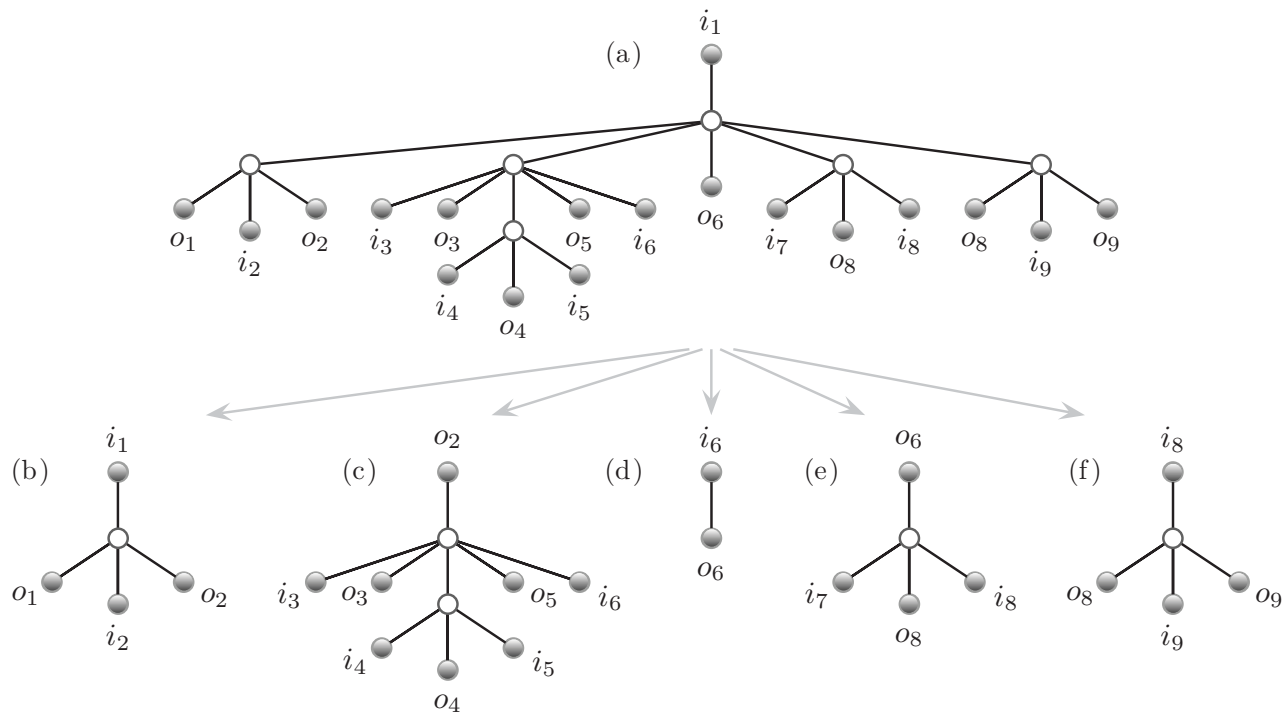

FIG. 6. The tree shown in (a) is cut at its top node (of degree 6) such that the trees (b)-(f) are created. Note that to complete the five new trees, we need to add an additional four new links and leaves and that the trees (c) and (e) in the even positions have the incoming and outgoing channels reversed.

vertices of the tree that correspond to encounters will be called "nodes" to distinguish them from the vertices of degree 1, which correspond to the incoming and outgoing channels and which will be called "leaves." The total number of nodes is $V=\sum_{l>1} v_{l}$ and the number of leaves is $2 n$, where $n$ is the order of the correlation function $C(\epsilon, n)$ to which the trees contribute. Defining $L=\sum_{l>1} l v_{l}$, we can express $n$ as $n=(L-V+1)$. Note that the total number of links is $L+n$, which can be seen as $l$ links trailing each $l$-encounter plus another $n$ from the incoming channels. For example, the $2^{1} 3^{1}$ tree in Fig. $4 \mathrm{i}$ has $L=5, V=2$ and contributes to the $n=4$ correlation function. We always draw the tree with the leaves ordered $i_{1}, o_{1}, \ldots, i_{n}, o_{n}$ in anticlockwise direction. This fixes the layout of the tree in the plane, thus the name "rooted plane trees." 66

From the start tree, we can also move some encounters into the lead(s) and it is easy to read off when this is possible. If an $l$-encounter (node of degree $2 l$ ) is adjacent to exactly $l$ leaves with label $i$, it may " $i$-touch" the lead, i.e., the electron trajectories have an encounter upon entering the system and the corresponding incoming channels coincide. Likewise if a $2 l$-node is adjacent to $l o$-leaves, it may " $O$-touch" the lead. For example, in Fig. 4(i), the top node has degree 6, is adjacent to $3 i$-leaves (including the root), and can $i$-touch the lead as in Figs. 5(b) and 5(e). The lower encounter can $o$-touch as in Figs. 5(a) and 5(d). In addition, both encounters can touch the lead to create Figs. 5(c) and 5(f).

Semiclassically, we add the contributions of all the possible trajectory structures (or trees), and the contribution of each is made up by multiplying the contributions of its constituent parts (links, encounters, and leaves). First we count the orders of the number of channels $N$. As mentioned in Ref. 47 (see also Sec. IV below), the multiplicative contribution of each encounter or leaf is of order $N$, and each link gives a contribution of order $1 / N$. Together with the overall factor of $1 / N$, see equation (16), the total power of $1 / N$ is $\gamma$, the cyclicity of the diagram. Since our diagrams must be connected, the smallest cyclicity is $\gamma=0$ if the diagram is a tree. The trees can be generated recursively, since by cutting a tree at the top node of degree $2 l$ (after the root) we obtain $2 l-1$ subtrees, as illustrated in Fig. 6 .

To track the trees and their nodes, the generating function $F\left(\boldsymbol{x}, z_{i}, z_{\boldsymbol{o}}\right)$ was introduced, ${ }^{47}$ where the powers of $x_{l}$ enumerate the number of $l$-encounters, $z_{i, l}$ enumerate the number of $l$-encounters that $i$-touch the lead, and $z_{o, l}$ enumerate the number of $l$-encounters that $o$-touch the lead.

Later, we will assign values to these variables that will produce the correct semiclassical contributions of the trees. Note that the contributions of the links and leaves will be absorbed into the contributions of the nodes, hence we do not directly enumerate the links in the generating function $F$. Inside $F$ we want to add all the possible trees and for each have a multiplicative contribution of its nodes. For example, the tree in Fig. 4(i) and its relatives in Fig. 5 would contribute

$x_{3} x_{2}+z_{i, 3} x_{2}+x_{3} z_{o, 2}+z_{i, 3} z_{o, 2}=\left(x_{3}+z_{i, 3}\right)\left(x_{2}+z_{o, 2}\right)$.

A technical difficulty is that the top node may (if there are no further nodes) be able to both $i$-touch and $o$-touch, but clearly not at the same time. An auxiliary generating function $f=$ $f\left(\boldsymbol{x}, z_{i}, z_{o}\right)$ is thus introduced with the restriction that the top node is not allowed to $i$-touch the lead. We denote by "empty" a tree that contains no encounter nodes [like Fig. 6(d)]. An empty tree is assigned the value 1 [i.e., $f(0)=1$ ] to not affect the multiplicative factors. To obtain a recursion for $f$, we separate the tree into its top node of degree $2 l$ and $2 l-1$ subtrees as in Fig. 6. As can be seen from the figure, $l$ of the new trees (in the odd positions from left to right) start with an incoming channel, while the remaining $l-1$ even-numbered subtrees start with an outgoing channel, and correspond to a tree with the $i$ 's and $o$ 's are reversed. For these we use the generating function $\hat{f}$, where the roles of the $z$ variables corresponding to leaves of one type are switched so $\hat{f}=f\left(\boldsymbol{x}, z_{o}, z_{i}\right)$. The tree 
then has the contribution of the top node times that of all the subtrees giving $x_{l} f^{l} \hat{f}^{l-1}$.

The top node may also $o$-touch the lead, but for this to happen all the odd-numbered subtrees must be empty (i.e., they must contain no further nodes and end directly in an outgoing channel). When this happens, we just get the contribution of $z_{o, l}$ times that of the $l-1$ even subtrees: $z_{o, l} \hat{f}^{l-1}$. In total, we have

$$
f=1+\sum_{l=2}^{\infty}\left[x_{l} f^{l} \hat{f}^{l-1}+z_{o, l} \hat{f}^{l-1}\right]
$$

and similarly

$$
\hat{f}=1+\sum_{l=2}^{\infty}\left[x_{l} \hat{f}^{l} f^{l-1}+z_{i, l} f^{l-1}\right] .
$$

For $F$ we then reallow the top node to $i$-touch the lead, which means that the even subtrees must be empty and a contribution of $z_{i, l} f^{l}$, giving

$$
F=f+\sum_{l=2}^{\infty} z_{i, l} f^{l}=\sum_{l=1}^{\infty} z_{i, l} f^{l},
$$

if we let $z_{i, 1}=1$ (and also $z_{o, 1}=1$ for symmetry). Picking an $o$-leaf as the root instead of an $i$-leaf should lead to the same trees and contributions, so $F$ should be symmetric upon swapping $z_{i}$ with $z_{o}$ and $f$ with $\hat{f}$. These recursions enumerate all possible trees (which represent all diagrams at leading order in inverse channel number), and we now turn to evaluating their contributions to the correlation functions $C(\epsilon, n)$.

\section{DENSITY OF STATES WITH A SINGLE LEAD}

To calculate the contribution of each diagram, Refs. 55-57 used the ergodicity of the classical motion to estimate how often the electron trajectories are likely to approach each other and have encounters. Combined with the sum rule 55,67 to deal with the stability amplitudes, Ref. 56 showed that the semiclassical contribution can be written as a product of integrals over the durations of the links and the stable and unstable separations of the stretches in each encounter. One ingredient is the survival probability that the electron trajectories remain inside the system (these are followed by the holes, whose conditional survival probability is then 1), which classically decays exponentially with their length and the decay rate $1 / \tau_{\mathrm{d}}=N / T_{\mathrm{H}}$. A small but important effect is that the small size of the encounters means the trajectories are close enough to remain inside the system or escape (hit the lead) together, so only one traversal of each encounter needs to be counted in the total survival probability,

$$
\exp \left(-\frac{N}{T_{\mathrm{H}}} t_{\mathrm{x}}\right), \quad t_{\mathrm{x}}=\sum_{i=1}^{L+n} t_{i}+\sum_{\alpha=1}^{V} t_{\alpha},
$$

where the $t_{i}$ are the durations of the $(n+L)$ link stretches and $t_{\alpha}$ the durations of the $V$ encounters so that the exposure time $t_{\mathrm{x}}$ is shorter than the total trajectory time (which includes $l$ copies of each $l$-encounter).

As reviewed in Ref. 57, the integrals over the links and the encounters (with their action differences) lead to simple diagrammatic rules whereby each link provides a factor of $T_{\mathrm{H}} /[N(1-\mathrm{i} \epsilon)]$, and each $l$-encounter inside the cavity provides a factor of $-N(1-\mathrm{i} l \epsilon) / T_{\mathrm{H}}^{l}$, with the $(1-\mathrm{i} l \epsilon)$ deriving from the difference between the exposure time and the total trajectory time. Recalling the prefactor in (18) and that $L$ is the total number of links in the encounters, it is clear that all the Heisenberg times cancel. The channel number factor $N^{-2 n}$ from these rules and the prefactor (with $n=L-V+1$ ) cancel with the sum over the channels in (18) as each of the $2 n$ channels can be chosen from the $N$ possible channels (to leading order).

With this simplification, each link gives $(1-\mathrm{i} \epsilon)^{-1}$, each encounter $-(1-\mathrm{i} l \epsilon)$, and each leaf a factor of 1 . To absorb the link contributions into those of the encounters (nodes), we recall that the number of links is $n+\sum_{\alpha=1}^{V} l_{\alpha}$, where $\alpha$ labels the $V$ different encounters. Therefore, the total contribution factorizes as

$$
\frac{1}{(1-\mathrm{i} \epsilon)^{n}} \prod_{\alpha=1}^{V} \frac{-\left(1-\mathrm{i} l_{\alpha} \epsilon\right)}{(1-\mathrm{i} \epsilon)^{l_{\alpha}}} .
$$

Moving an $l$-encounter into the lead, as in Fig. 5, means losing that encounter, $l$ links, and combining $l$ channels, so we just remove that encounter from the product above (or give it a factor 1 instead).

\section{A. Generating function}

Putting these diagrammatic rules into the recursions in Sec. III A then simply means setting

$$
x_{l}=\frac{-(1-\mathrm{i} l \epsilon)}{(1-\mathrm{i} \epsilon)^{l}} \tilde{r}^{l-1}, \quad z_{i, l}=z_{o, l}=\tilde{r}^{l-1},
$$

where we additionally include powers of $\tilde{r}$ to track the order of the trees and later generate the semiclassical correlation functions. The total power of $\tilde{r}$ of any tree is $\sum_{l>1}(l-1) v_{l}=$ $L-V=n-1$. To get the required prefactor of $(1-\mathrm{i} \epsilon)^{-n}$ in (24), we can then make the change of variable

$$
f=g(1-\mathrm{i} \epsilon), \quad \tilde{r}=\frac{r}{1-\mathrm{i} \epsilon},
$$

so that the recursion relation (20) becomes

$$
g(1-\mathrm{i} \epsilon)=1-\sum_{l=2}^{\infty} r^{l-1} g^{l} \hat{g}^{l-1}(1-\mathrm{i} l \epsilon)+\sum_{l=2}^{\infty} r^{l-1} \hat{g}^{l-1},
$$

and similarly for $\hat{g}$. Using geometric sums (the first two terms are the $l=1$ terms of the sums), this is

$$
\frac{g}{1-r g \hat{g}}=\frac{\mathrm{i} \epsilon g}{(1-r g \hat{g})^{2}}+\frac{1}{1-r \hat{g}} \text {. }
$$

We note that since $\hat{f}$ is obtained from $f$ by swapping $z_{i}$ and $z_{o}$ and in our substitution (25) $\boldsymbol{z}_{i}=\boldsymbol{z}_{o}$, the functions $\hat{f}$ and $f$ are equal. Taking the numerator of the equation above and substituting $\hat{g}=g$ leads to

$$
g-\frac{1}{1-\mathrm{i} \epsilon}=\frac{r g^{2}}{1-\mathrm{i} \epsilon}[g-1-\mathrm{i} \epsilon] .
$$


To obtain the desired generating function of the semiclassical correlation functions, we set $F=G(1-\mathrm{i} \epsilon)$ in (22), along with the other substitutions in (25) and (26),

$$
G(\epsilon, r)=\frac{g}{1-r g}, \quad G(\epsilon, r)=\sum_{n=1}^{\infty} r^{n-1} C(\epsilon, n),
$$

so that by expanding $g$ and hence $G$ in powers of $r$, we obtain all the correlation functions $C(\epsilon, n)$. This can be simplified by rearranging (30) and substituting into (29) to get the cubic for $G$ directly,

$r(r-1)^{2} G^{3}+r(3 r+\mathrm{i} \epsilon-3) G^{2}+(3 r+\mathrm{i} \epsilon-1) G+1=0$.

\section{B. Density of states}

The density of states of a chaotic Andreev billiard with one superconducting lead (15) can be rewritten as

$$
d(\epsilon)=1-2 \operatorname{Im} \frac{\partial}{\partial \epsilon} \sum_{n=1}^{\infty} \frac{(-1)^{n-1} C(\epsilon, n)}{n},
$$

where without the $1 / n$ the sum would just be $G(\epsilon,-1)$ in view of (30). To obtain the $1 / n$, we can formally integrate to obtain a new generating function $H(\epsilon, r)$,

$$
\begin{aligned}
& H(\epsilon, r)=\frac{1}{\mathrm{i} r} \frac{\partial}{\partial \epsilon} \int G(\epsilon, r) \mathrm{dr}, \\
& H(\epsilon, r)=\sum_{n=1}^{\infty} \frac{r^{n-1}}{\mathrm{i} n} \frac{\partial C(\epsilon, n)}{\partial \epsilon},
\end{aligned}
$$

so the density of states is given simply by

$$
d(\epsilon)=1-2 \operatorname{Re} H(\epsilon,-1) .
$$

To evaluate the sum in (32), we now need to integrate the solutions of (31) with respect to $r$ and differentiate with respect to $\epsilon$. Since $G$ is an algebraic generating function, i.e., the solution of an algebraic equation, the derivative of $G$ with respect to $\epsilon$ is also an algebraic generating function. ${ }^{68}$ However, this is not generally true for integration, which can be seen from a simple example of $f=1 / x$, which is a root of an algebraic equation, unlike the integral of $f$. Solving equation (31) explicitly and integrating the result is also technically challenging, due to the complicated structure of the solutions of the cubic equations. Even if it were possible, this approach would fail in the presence of magnetic field, when $G$ is a solution of a quintic equation, see Sec. IV D, or in the presence of a phase difference between two superconductors.

The approach we took is to conjecture that $H(\epsilon, r)$ is given by an algebraic equation, perform a computer-aided search over equations with polynomial coefficients, and then prove the answer by differentiating appropriately. We found that

$$
\begin{aligned}
& (\epsilon r)^{2}(1-r) H^{3}+\mathrm{i} \epsilon r[r(\mathrm{i} \epsilon-2)+2(1-\mathrm{i} \epsilon)] H^{2} \\
& \quad+\left[r(1-2 \mathrm{i} \epsilon)-(1-\mathrm{i} \epsilon)^{2}\right] H+1=0,
\end{aligned}
$$

when expanded in powers of $r$, agrees for a range of values of $n$ with the expansion of (33) derived from the correlation functions obtained from (31). To show that (35) agrees with
(33) to all orders in $r$, we use a differentiation algorithm to find an equation for the intermediate generating function,

$$
\begin{aligned}
& I(\epsilon, r)=\frac{1}{\mathrm{i}} \frac{\partial G(\epsilon, r)}{\partial \epsilon}=\frac{\partial[r H(\epsilon, r)]}{\partial r}, \\
& I(\epsilon, r)=\sum_{n=1}^{\infty} \frac{r^{n-1}}{\mathrm{i}} \frac{\partial C(\epsilon, n)}{\partial \epsilon},
\end{aligned}
$$

both starting from (31) and from (35) and verifying that the two answers agree.

The differentiation algorithm starts with the algebraic equation for a formal power series $\eta$ in the variable $x$, which satisfies an equation of the form

$$
\Phi(x, \eta):=p_{0}(x)+p_{1}(x) \eta+\cdots+p_{m}(x) \eta^{m}=0,
$$

where $p_{0}(x), \ldots, p_{m}(x)$ are some polynomials, not all of them zero. The aim is to find an equation satisfied by $\xi=\mathrm{d} \eta / \mathrm{d} x$, of the form

$$
q_{0}(x)+q_{1}(x) \xi+\cdots+q_{m}(x) \xi^{m}=0,
$$

where $q_{0}(x), \ldots, q_{m}(x)$ are polynomials. Differentiating (37) implicitly yields

$$
\xi=-\frac{\partial \Phi(x, \eta)}{\partial x}\left(\frac{\partial \Phi(x, \eta)}{\partial \eta}\right)^{-1}=\frac{P(\eta, x)}{Q(\eta, x)},
$$

where $P$ and $Q$ are again polynomial. After substituting this expression into the algebraic equation for $\xi$ and bringing everything to the common denominator, we get

$$
\begin{gathered}
q_{0}(x) Q^{m}(x, \eta)+q_{1}(x) P(x, \eta) Q^{m-1}(x, \eta) \\
+\cdots+q_{m}(x) P^{m}(x, \eta)=0 .
\end{gathered}
$$

However, this equation should only be satisfied modulo the polynomial $\Phi(x, \eta)$. Namely, we use polynomial division and substitute $P^{j}(x, \eta) Q^{m-j}(x, \eta)=$ $T(x, \eta) \Phi(x, \eta)+R_{j}(x, \eta)$ into (40). Using (37), we arrive at

$$
q_{0}(x) R_{0}(x, \eta)+q_{1}(x) R_{1}(x, \eta)+\cdots+q_{m}(x) R_{m}(x, \eta)=0 .
$$

The polynomials $R_{j}$ are of degree of $m-1$ in $\eta$. Treating (41) as an identity with respect to $\eta$, we thus obtain $m$ linear equations on the coefficients $q_{j}$. Solving those, we obtain $q_{j}$ as rational functions of $x$, and multiplying them by their common denominator gives the algebraic equation for $\xi$.

Performing this algorithm on $G$ from (31), with $x=\mathrm{i} \epsilon$, and on $r H$ from (35), with $x=r$, leads to the same equation, given as (A1) in the Appendix, for the intermediate function defined in (36), and therefore proves the validity of the equation (35). Setting $\epsilon=0$ in (35) then shows that $\left.\frac{\partial C(\epsilon, n)}{\partial \epsilon}\right|_{\epsilon=0}=$ in as mentioned in Sec. II B. To compare the final result (34) with the RMT prediction, we can substitute $H(\epsilon,-1)=[-\mathrm{i} W(\epsilon)+1] / 2$ into (35). The density of states is then given in terms of $W$ as $d(\epsilon)=-\operatorname{Im} W(\epsilon)$. The equation for $W$ simplifies to the RMT result (12), and the density of states then reads ${ }^{27}$

$$
d(\epsilon)= \begin{cases}0, & \epsilon \leqslant 2\left(\frac{\sqrt{5}-1}{2}\right)^{5 / 2}, \\ \frac{\sqrt{3}}{6 \epsilon}\left[Q_{+}(\epsilon)-Q_{-}(\epsilon)\right], & \epsilon>2\left(\frac{\sqrt{5}-1}{2}\right)^{5 / 2},\end{cases}
$$



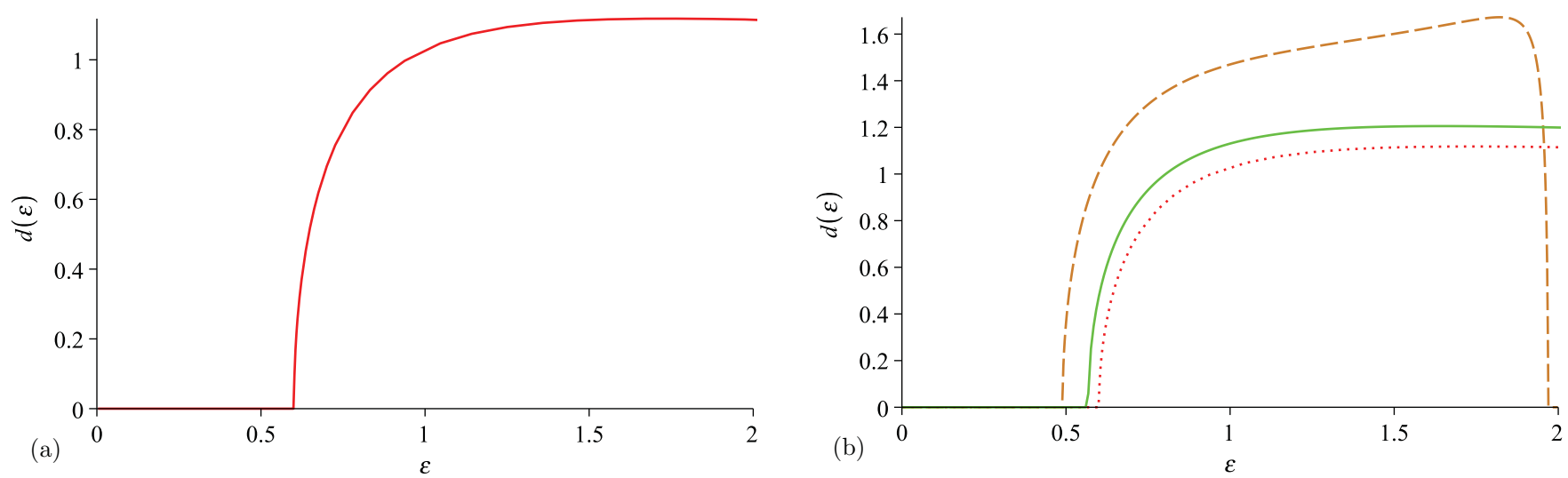

FIG. 7. (Color online) (a) The density of states of a chaotic quantum dot coupled to a single superconductor at $E \ll \Delta$. (b) The density of states with a finite bulk superconducting gap $\Delta=2 E_{T}$ (dashed line) and $\Delta=8 E_{T}$ (solid line) compared to the previous case in (a) with $\Delta \rightarrow \infty$ (dotted line).

where $Q_{ \pm}(\epsilon)=\left(8-36 \epsilon^{2} \pm 3 \epsilon \sqrt{3 \epsilon^{4}+132 \epsilon^{2}-48}\right)^{1 / 3}$. This result is plotted in Fig. 7(a) and shows the hard gap extending up to around $0.6 E_{T}$.

\section{Small bulk superconducting gap}

The calculation of the density of states above used the approximation that the energy was well below the bulk superconductor gap, $E \ll \Delta$ or $\epsilon \ll \delta$ (for $\delta=\Delta / E_{T}$ ), so that the phase shift at each Andreev reflection was $\arccos (\epsilon / \delta) \approx$ $\pi / 2$. For higher energies or smaller superconducting gaps, however, the density of states should be modified ${ }^{69}$ to

$$
d(\epsilon)=1+\operatorname{Re} \frac{2}{\sqrt{\delta^{2}-\epsilon^{2}}}+2 \operatorname{Im} \sum_{n=1}^{\infty} \frac{\partial}{\partial \epsilon}\left[\frac{\alpha(\epsilon)^{2 n} C(\epsilon, n)}{n}\right],
$$

where $\alpha(\epsilon)=\delta /\left(\epsilon+\mathrm{i} \sqrt{\delta^{2}-\epsilon^{2}}\right)$ as in (3). When taking the energy derivative in the sum in (43), we can split the result into two sums and hence two contributions to the density of states

$$
\begin{aligned}
d(\epsilon)= & 1+2 \operatorname{Im} \sum_{n=1}^{\infty} \frac{\alpha(\epsilon)^{2 n}}{n} \frac{\partial C(\epsilon, n)}{\partial \epsilon} \\
& +\operatorname{Re} \frac{2}{\sqrt{\delta^{2}-\epsilon^{2}}}\left[1+2 \sum_{n=1}^{\infty} \frac{\alpha(\epsilon)^{2 n} C(\epsilon, n)}{n}\right] .
\end{aligned}
$$

Here the first term, which comes from applying the energy derivative to $C(\epsilon, n)$, gives an analogous contribution to the case $E \ll \Delta$ but with $r=\alpha^{2}$ instead of -1 and involving $H\left(\epsilon, \alpha^{2}\right)$ from (33) and (35). The second term in (44) comes from the energy derivative of $\alpha^{2 n}$ and can be written using $G\left(\epsilon, \alpha^{2}\right)$ from (30) and (31):

$$
\begin{aligned}
d(\epsilon)= & \operatorname{Re}\left[1+2 \alpha^{2} H\left(\epsilon, \alpha^{2}\right)\right] \\
& +\operatorname{Re} \frac{2}{\sqrt{\delta^{2}-\epsilon^{2}}}\left[1+2 \alpha^{2} G\left(\epsilon, \alpha^{2}\right)\right] .
\end{aligned}
$$

The effect of a finite bulk superconducting gap on the hard gap in the density of states of the Andreev billiard is fairly small. For example, as shown in Fig. 7(b), even for $\delta=\Delta / E_{T}=2$ the width just shrinks to around $0.5 E_{T}$. For $\delta=2$, the shape of the density of states is changed somewhat (less so for $\delta=8$ ) and we can see just before $\epsilon=2$ it vanishes again giving a second thin gap. This gap, and even the way we can separate the density of states into the two terms in (45), foreshadows the effects of the Ehrenfest time (in Sec. VI). For energies above the bulk superconducting gap $(\epsilon>\delta)$, we see a thin singular peak from the $\sqrt{\delta^{2}-\epsilon^{2}}$ that quickly tends to the density of states of an Andreev billiard with an infinite superconducting gap as the energy becomes larger.

\section{Magnetic field}

If a magnetic field is present, the time-reversal symmetry is broken and we wish to treat this transition semiclassically as in Refs. 64 and 70. Note that since for the leading-order diagrams each stretch is traversed in opposite directions by an electron and a hole, we are effectively considering the same situation as for parametric correlations. ${ }^{71,72}$ Either way, the idea behind the treatment is that the classically small magnetic field affects the classical trajectories very little, but adds many essentially random small perturbations to the action. The sum of these fluctuations is approximated using the central limit theorem, and leads to an exponential damping, so the links now provide a factor of $T_{\mathrm{H}} / N(1-\mathrm{i} \epsilon+b)$. The parameter $b$ is related to the magnetic field via $b=\left(\Phi / \Phi_{\mathrm{c}}\right)^{2}$ as in Sec. II A. For an $l$-encounter, however, as the stretches are correlated and affected by the magnetic field in the same way, the variance of the random fluctuations of all the stretches is $l^{2}$ that of a single stretch. Hence each encounter now contributes $N(1-$ $\left.\mathrm{i} l \epsilon+l^{2} b\right) / T_{\mathrm{H}}^{l}$ and again the correlation inside the encounters leads to a small but important effect.

Similarly to the treatment without the magnetic field above, we can put these contributions into the recursions in Sec. III A by setting

$$
x_{l}=\frac{-\left(1-\mathrm{i} l \epsilon+l^{2} b\right)}{(1-\mathrm{i} \epsilon+b)^{l}} \tilde{r}^{l-1}, \quad z_{i, l}=z_{o, l}=\tilde{r}^{l-1},
$$

and

$$
f=g(1-\mathrm{i} \epsilon+b), \quad \tilde{r}=\frac{r}{1-\mathrm{i} \epsilon+b} .
$$


The intermediate generating function is then given by the implicit equation

$$
\begin{aligned}
& -r^{2} g^{5}+(1+\mathrm{i} \epsilon+b) r^{2} g^{4}+(2-\mathrm{i} \epsilon-b) r g^{3} \\
& -(2+\mathrm{i} \epsilon-b) r g^{2}-(1-\mathrm{i} \epsilon+b) g+1=0,
\end{aligned}
$$

and the generating function $G(\epsilon, b, r)$ of the magnetic-fielddependent correlation functions $C(\epsilon, b, n)$, which is still connected to $g$ via $G=g /(1-r g)$, is given by

$$
\begin{aligned}
& r^{2}(r-1)^{3} G^{5} \\
& \quad+\left(\mathrm{i} \epsilon r-\mathrm{i} \epsilon+5 r^{2}-10 r+5-b r-b\right) r^{2} G^{4} \\
& \quad+\left(3 \mathrm{i} \epsilon r-\mathrm{i} \epsilon+10 r^{2}-12 r+2-3 b r-b\right) r G^{3} \\
& \quad+(3 \mathrm{i} \epsilon+10 r-6-3 b) r G^{2} \\
& \quad-(1-5 r-\mathrm{i} \epsilon+b) G+1=0 .
\end{aligned}
$$

Removing the magnetic field by setting $b=0$ reduces both of these equations (after factorizing) to the previous results (29) and (31). Next we again search for and verify an algebraic equation for $H(\epsilon, b, r)=1 /(\mathrm{i} r) \int[\partial G(\epsilon, b, r) / \partial \epsilon] \mathrm{d} r$, though the higher order makes this slightly more complicated, finding

$$
\begin{aligned}
& 4 b^{2} r^{4}(r-1) H^{5}+4 b r^{3}[\mathrm{i} \epsilon-3 b+r(2 b-\mathrm{i} \epsilon)] H^{4} \\
& \quad+r^{2}\left[\epsilon^{2}(1-r)+2 \mathrm{i} \epsilon b(5-3 r)-b(13 b+4)\right. \\
& \quad+b r(5 b+4)] H^{3}+r\{2(\mathrm{i} \epsilon-3 b)(1-\mathrm{i} \epsilon+b) \\
& \quad+r\left[(1-\mathrm{i} \epsilon+b)^{2}+4 b-1\right] H^{2}-\left[(1-\mathrm{i} \epsilon+b)^{2}\right. \\
& \quad-r(1-2 \mathrm{i} \epsilon+2 b)]\} H+1=0 .
\end{aligned}
$$

To check the agreement with the RMT result, we substitute $H(\epsilon, b,-1)=[-\mathrm{i} W(\epsilon, b)+1] / 2$ into (50). This leads to

$$
\begin{gathered}
b^{2} W^{5}-2 b \epsilon W^{4}-\left(4 b-b^{2}-\epsilon^{2}\right) W^{3}+2(2-b) \epsilon W^{2} \\
+\left(4-4 b+\epsilon^{2}\right) W+4 \epsilon=0,
\end{gathered}
$$

which corresponds to the RMT result (11) with no phase $(\phi=0)$. The density of states calculated from this equation is shown in Fig. 8 for different values of $b$. The gap reduces for increasing $b$, closes exactly at the critical flux $(b=1)$, and the density of states becomes flat (at 1) as $b \rightarrow \infty$.

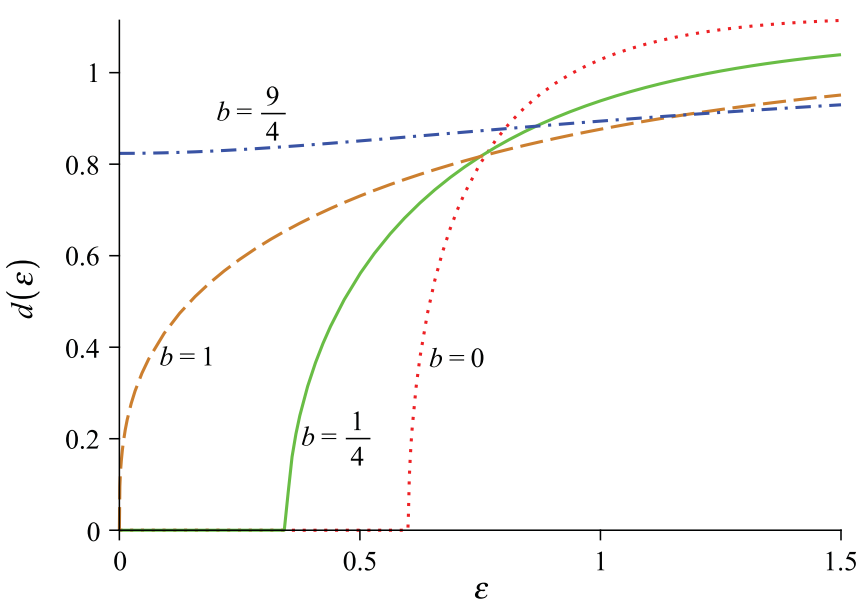

FIG. 8. (Color online) The effect of a time-reversal symmetrybreaking magnetic field on the density of states of a chaotic Andreev billiard with a single superconducting lead for $b=0$ (dotted line), $b=1 / 4$ (solid line), $b=1$ (dashed line), and $b=9 / 4$ (dashed dotted line).

\section{DENSITY OF STATES WITH TWO LEADS}

Next we consider a classically chaotic quantum dot connected to two superconductors with $N_{1}$ and $N_{2}$ channels, respectively, and a phase difference $\phi$, as depicted in Fig. 2(a). The density of states, as in Sec. II A and Refs. 35 and 69, can then be reduced to equation (15) but with

$$
C(\epsilon, \phi, n)=\frac{1}{N} \operatorname{Tr}\left[S^{*}\left(-\frac{\epsilon \hbar}{2 \tau_{\mathrm{d}}}\right) \mathrm{e}^{-\mathrm{i} \tilde{\phi}} S\left(+\frac{\epsilon \hbar}{2 \tau_{\mathrm{d}}}\right) \mathrm{e}^{\mathrm{i} \tilde{\phi}}\right]^{n}
$$

where $\tilde{\phi}$ is again a diagonal matrix whose first $N_{1}$ elements from the first superconductor $S_{1}$ are $\phi / 2$ and the remaining $N_{2}$ elements from $\mathrm{S}_{2}$ are $-\phi / 2$. Note that the case $\phi=0$ corresponds to the previous case of a single superconductor with $N=N_{1}+N_{2}$ channels. When we substitute the semiclassical approximation for the scattering matrix (17) into (52), and especially if we write the scattering matrix in terms of its reflection and transmission subblocks, the effect of the superconductors' phase difference becomes simple. Namely, each electron (unprimed) trajectory that starts in lead 1 and ends in lead 2 picks up the phase factor $\exp (-\mathrm{i} \phi)$, while each unprimed trajectory going from lead 2 to lead 1 receives the factor $\exp (\mathrm{i} \phi)$. Reflection trajectories that start and end in the same lead have no additional phase factor, as depicted in Fig. 9. Since exchanging the leads gives the opposite phase, we expect the solution to be symmetric if we simultaneously exchange $N_{1}$ with $N_{2}$ and change $\phi$ to $-\phi$.

As these factors are multiplicative, we can equivalently say that each electron trajectory leaving superconductor 1 or 2 picks up $\exp (-\mathrm{i} \phi / 2)$ or $\exp (\mathrm{i} \phi / 2)$, while each one entering lead 1 or 2 picks up $\exp (\mathrm{i} \phi / 2)$ or $\exp (-\mathrm{i} \phi / 2)$. To include these factors in our semiclassical diagrams, we can simply remember that in our tree recursions in Sec. III A, the channels we designated as "incoming" channels have electrons leaving them while electrons always enter the outgoing channels. Each incoming channel [in the original channel sum in (18)] can still come from the $N$ possible channels, but with the trajectory leaving it now provides the factor $N_{1} \exp (-\mathrm{i} \phi / 2)+$ $N_{2} \exp (\mathrm{i} \phi / 2)$. Similarly, each outgoing channel now provides the complex conjugate of this factor. Recalling the power of

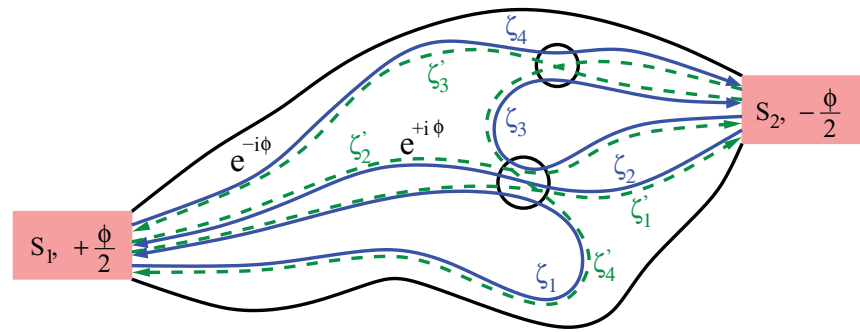

FIG. 9. (Color online) The paths may start and end in either of the two leads as shown. $\zeta_{4}$ as it travels from lead 1 to lead 2 obtains a phase factor $\exp (-\mathrm{i} \phi), \zeta_{2}$ traveling back contributes $\exp (\mathrm{i} \phi)$, while the others do not contribute any phase. The encounters are again marked by circles and $S_{1}$ and $S_{2}$ denote the two superconducting leads at the corresponding superconducting phases $\pm \phi / 2$. This diagram is equivalent to the one in Fig. 4(f). 
$N^{-2 n}$ coming from the links and encounters, we can update the contribution of each diagram or tree (24) to

$$
\begin{aligned}
& \frac{\left(N_{1} \mathrm{e}^{-\frac{\mathrm{i} \phi}{2}}+N_{2} \mathrm{e}^{\frac{\mathrm{i} \phi}{2}}\right)^{n}\left(N_{1} \mathrm{e}^{\mathrm{i} \phi}+N_{2} \mathrm{e}^{-\frac{\mathrm{i} \phi}{2}}\right)^{n}}{N^{2 n}(1-\mathrm{i} \epsilon)^{n}} \\
& \times \prod_{\alpha=1}^{V} \frac{-\left(1-\mathrm{i} l_{\alpha} \epsilon\right)}{(1-\mathrm{i} \epsilon)^{l_{\alpha}}} .
\end{aligned}
$$

However, moving an $l$-encounter into lead 1 means combining $l$ incoming channels, $l$ links, and the encounter itself. These combined incoming channels, with $l$ electron trajectories leaving, will now only give the factor $N_{1} \exp (-\mathrm{i} l \phi / 2)+$ $N_{2} \exp (\mathrm{i} l \phi / 2)$, where the important difference is that $l$ is inside the exponents. We therefore make the replacement

$$
\frac{\left(N_{1} \mathrm{e}^{-\frac{\mathrm{i} \phi}{2}}+N_{2} \mathrm{e}^{\mathrm{i} \phi}\right)^{l}}{N^{l}} \rightarrow \frac{\left(N_{1} \mathrm{e}^{-\frac{\mathrm{i} l \phi}{2}}+N_{2} \mathrm{e}^{\mathrm{i} / \phi}\right)}{N}
$$

as well as removing the encounter from (53). Similarly, when we move the encounter into the outgoing leads, we take the complex conjugate of (54).

To mimic these effects in the semiclassical recursions, we can set

$$
\begin{gathered}
x_{l}=\frac{-(1-\mathrm{i} l \epsilon)}{(1-\mathrm{i} \epsilon)^{l}} \tilde{r}^{l-1}, \quad \beta=\frac{\left(N_{1} \mathrm{e}^{-\frac{\mathrm{i} \phi}{2}}+N_{2} \mathrm{e}^{\frac{\mathrm{i} \phi}{2}}\right)}{N}, \\
z_{i, l}=\frac{\left(N_{1} \mathrm{e}^{-\frac{\mathrm{i} \phi}{2}}+N_{2} \mathrm{e}^{\frac{\mathrm{i} l \phi}{2}}\right)}{N \beta^{l}} \tilde{r}^{l-1}, \\
z_{o, l}=\frac{\left(N_{1} \mathrm{e}^{\mathrm{i} l \phi}+N_{2} \mathrm{e}^{-\frac{\mathrm{i} l \phi}{2}}\right)}{N\left(\beta^{*}\right)^{l}} \tilde{r}^{l-1}, \\
f=g \frac{(1-\mathrm{i} \epsilon)}{\beta \beta^{*}}, \quad \tilde{r}=r \frac{\beta \beta^{*}}{(1-\mathrm{i} \epsilon)},
\end{gathered}
$$

in Sec. III A. Including these substitutions in the recursion relation (20) and summing, we obtain

$$
\begin{aligned}
\frac{g}{\beta \beta^{*}-r g \hat{g}}= & \frac{\mathrm{i} \epsilon \beta \beta^{*} g}{\left(\beta \beta^{*}-r g \hat{g}\right)^{2}}+\frac{N_{1}}{N} \frac{1}{\beta^{*} \mathrm{e}^{-\frac{\mathrm{i} \phi}{2}}-r \hat{g}} \\
& +\frac{N_{2}}{N} \frac{1}{\beta^{*} \mathrm{e}^{\frac{\mathrm{i} \phi}{2}}-r \hat{g}},
\end{aligned}
$$

and a similar equation from (21). The generating function of the correlation functions $C(\epsilon, \phi, n)$ is then given from (22) by

$$
G=\frac{N_{1}}{N} \frac{g}{\beta \mathrm{e}^{\mathrm{i} \phi} \frac{N_{2}}{2}-r g}+\frac{g}{N \mathrm{e}^{\frac{-\mathrm{i} \phi}{2}}-r g} .
$$

Returning to (58) and multiplying through by $\hat{g}$, we can see that the first two terms are symmetric in $g$ and $\hat{g}$. Combining the other two and taking the difference from the corresponding equation for $\hat{g}$, we have

$$
\frac{\hat{g}\left[\left(\beta^{*}\right)^{2}-r \hat{g}\right]}{\left(\beta^{*} \mathrm{e}^{-\frac{\mathrm{i} \phi}{2}}-r \hat{g}\right)\left(\beta^{*} \mathrm{e}^{\frac{\mathrm{i} \phi}{2}}-r \hat{g}\right)}=\frac{g\left[\beta^{2}-r g\right]}{\left(\beta \mathrm{e}^{\frac{\mathrm{i} \phi}{2}}-r g\right)\left(\beta \mathrm{e}^{-\frac{\mathrm{i} \phi}{2}}-r g\right)} .
$$

The resulting quadratic equation, when substituted back into (58), leads to a sixth-order equation for $g$. Note that the righthand side of (60) is [recalling (55) and that $N_{1}+N_{2}=N$ ] the same as (59), so it is clear that $G$ satisfies the required symmetry upon swapping the leads (i.e., swapping $N_{1}$ with $N_{2}$ and $\phi$ with $-\phi$ ).

\section{A. Equal leads}

To make the equations more manageable, we focus for now on the simpler case in which the leads have equal size and $N_{1}=N_{2}=N / 2$. Then $\beta=\cos (\phi / 2)$ is real and we can see from (60) or $z_{i}=z_{o}$ that $g=\hat{g}$ is a solution. Putting this simplification into (58), we can obtain the following quartic:

$$
\begin{gathered}
r^{2} g^{4}-r(1+r+\mathrm{i} \epsilon r) g^{3}+2 \mathrm{i} \epsilon \beta^{2} r g^{2} \\
+(1-\mathrm{i} \epsilon+r) \beta^{2} g-\beta^{4}=0 .
\end{gathered}
$$

We may also find an algebraic equation of fourth order for $G$ if we solve (59) for $g$ and substitute the solution

$$
g=\frac{\beta}{2} \frac{2 r \beta G+\beta-\sqrt{\beta^{2}+4 r G(1+r G)\left(\beta^{2}-1\right)}}{r(1+r G)}
$$

into (61). Note that we take the negative square root to agree with the previous result when the phase is 0 (i.e., $\beta=1$ ), though this sign does not affect the equation one finally finds for $G$. After the fourth-order equation for $G$ has been found, we can again search for and verify an equation for $H(\epsilon, \phi, r)=$ $1 /(\mathrm{i} r) \int[\partial G(\epsilon, \phi, r) / \partial \epsilon] \mathrm{d} r$,

$$
\begin{aligned}
\epsilon^{2} & r^{3}\left[1-2 r\left(2 \beta^{2}-1\right)+r^{2}\right] H^{4}+\mathrm{i} \epsilon r^{2}[2-3 \mathrm{i} \epsilon-4 r \\
& \left.\times(1-\mathrm{i} \epsilon)\left(2 \beta^{2}-1\right)+r^{2}(2-\mathrm{i} \epsilon)\right] H^{3}-r\left[1-4 \mathrm{i} \epsilon-3 \epsilon^{2}\right. \\
& \left.-2 r\left(1-3 \mathrm{i} \epsilon-\epsilon^{2}\right)\left(2 \beta^{2}-1\right)+r^{2}(1-2 \mathrm{i} \epsilon)\right] H^{2} \\
& -\left[(1-\mathrm{i} \epsilon)^{2}-2 r(1-\mathrm{i} \epsilon)\left(2 \beta^{2}-1\right)+r^{2}\right] H+\beta^{2}=0 .
\end{aligned}
$$

To see the agreement of our result with the RMT prediction, we again substitute $H(\epsilon, \phi,-1)=[-\mathrm{i} W(\epsilon, \phi)+1] / 2$ such that $d(\epsilon)=-\operatorname{Im} W(\epsilon, \phi)$. If we do so, we find

$$
\begin{aligned}
& \epsilon^{2} \beta^{2} W^{4}+4 \epsilon \beta^{2} W^{3}+\left(4 \beta^{2}-\epsilon^{2}+2 \epsilon^{2} \beta^{2}\right) W^{2} \\
& \quad+4 \epsilon \beta^{2} W-\epsilon^{2}+\epsilon^{2} \beta^{2}=0,
\end{aligned}
$$

which corresponds to (11) for zero magnetic field. Moreover, if the phase difference is zero (and $\beta=1$ ), we can take out the factor $W$ and recover (12).

Solving this equation yields the density of states. If we insert different values for the phase $\phi$, one finds that the hard gap in the density of states decreases with increasing phase difference while the density of states has a peak at the end of the gap that increases and becomes sharper with increasing phase. Finally, when the phase difference is equal to $\pi$, the gap closes and the peak vanishes so the density of states becomes identical to 1. This can all be seen in Fig. 10.

\section{B. Magnetic field}

In the presence of a magnetic field, we again have to change the diagrammatic rules as in Sec. IV D. Doing the calculation above with these modified diagrammatic rules leads to a sixthorder equation for $g$ :

$$
\begin{aligned}
& r^{3} g^{6}-r^{2}[1+r(1+\mathrm{i} \epsilon+b)] g^{5}-r^{2} \beta^{2}(1-2 \mathrm{i} \epsilon-2 b) g^{4} \\
& +r \beta^{2}[2-\mathrm{i} \epsilon-b+r(2+\mathrm{i} \epsilon-b)] g^{3}-r \beta^{4} \\
& \quad \times(1+2 \mathrm{i} \epsilon-2 b) g^{2}-\beta^{4}(1+r-\mathrm{i} \epsilon+b) g+\beta^{6}=0 .
\end{aligned}
$$




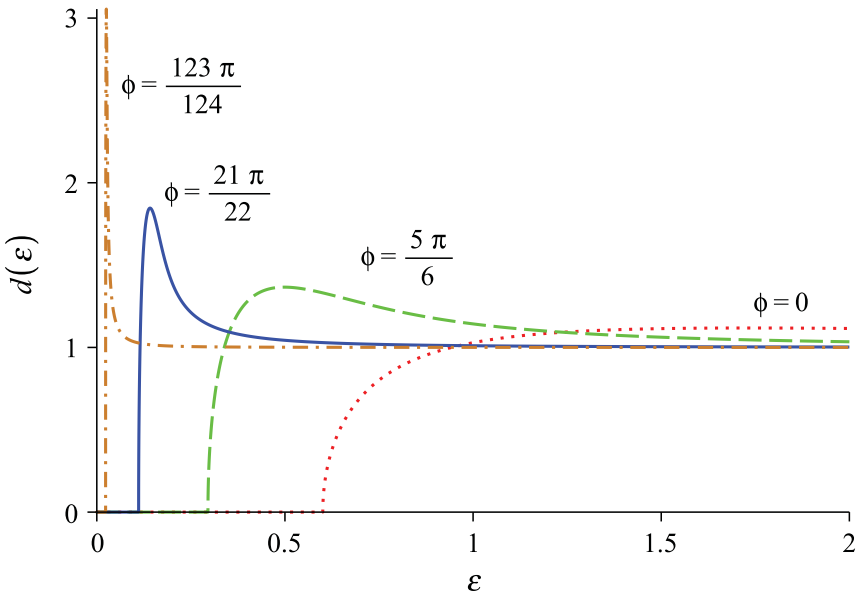

FIG. 10. (Color online) The density of states of a chaotic quantum dot coupled to two superconductors with the same numbers of channels and phase differences 0 (dotted line), $5 \pi / 6$ (solid line), $21 \pi / 22$ (dashed line), and $123 \pi / 124$ (dashed dotted line).

The relation (59) between $G$ and $g$ remains unchanged, and therefore we may find a sixth-order equation for $G$. We find the corresponding $H$, which is recorded as (A2) in the Appendix, using a computer search over sixth-order equations with polynomial (in $\epsilon, \phi, b$, and $r$ ) coefficients whose expansion in $r$ (33) matches the correlation functions calculated by expanding $G$. We note that for this order polynomial, it was not feasible (in terms of computational time and memory) to solve the equations resulting from the differentiation algorithm described in Sec. IV B and to find the intermediate generating function $I$ in all generality. However, we succeeded in finding a polynomial equation for $I$ that was satisfied by the derivatives of both $r H$ and $G$ for a large number of numerical values of the parameters $(\epsilon, \phi, b)$. For each parameter involved, the number of values checked was larger than the maximum degree of the parameter in the conjectured equation. While we cannot rule out the possibility that the true equation for $I$ has a higher order, given the large number of numerical values checked this is highly unlikely.

From $H$ we obtain the equation for $W(\epsilon, \phi, b)$,

$$
\begin{aligned}
& b^{2} \beta^{2} W^{6}-2 \epsilon b \beta^{2} W^{5}+\left(2 b^{2} \beta^{2}+\epsilon^{2} \beta^{2}-4 b \beta^{2}-b^{2}\right) W^{4} \\
& \quad+2\left(\epsilon b+2 \epsilon \beta^{2}-2 \epsilon b \beta^{2}\right) W^{3}+\left(4 \beta^{2}-b^{2}-\epsilon^{2}-4 b \beta^{2}\right. \\
& \left.\quad+b^{2} \beta^{2}+2 \epsilon^{2} \beta^{2}\right) W^{2}+2\left(\epsilon b+2 \epsilon \beta^{2}-\epsilon b \beta^{2}\right) W-\epsilon^{2} \\
& \quad+\epsilon^{2} \beta^{2}=0
\end{aligned}
$$

which corresponds exactly to the full RMT result (11) expanded.

As an example, the magnetic-field dependence of the density of states is shown at the phase difference of $5 \pi / 6$ in Fig. 11. As the magnetic field is increased, one finds a reduction of the gap, and the peak appearing for a phase difference $\phi>0$ vanishes again. Moreover, the higher the phase difference, the lower the magnetic field needed to close the gap. While for $\phi=0$ the gap closes at $b=1$, in the case of a phase difference of $5 \pi / 6$ one needs $b \approx 0.4096$ and for $\phi=21 \pi / 22$ a magnetic

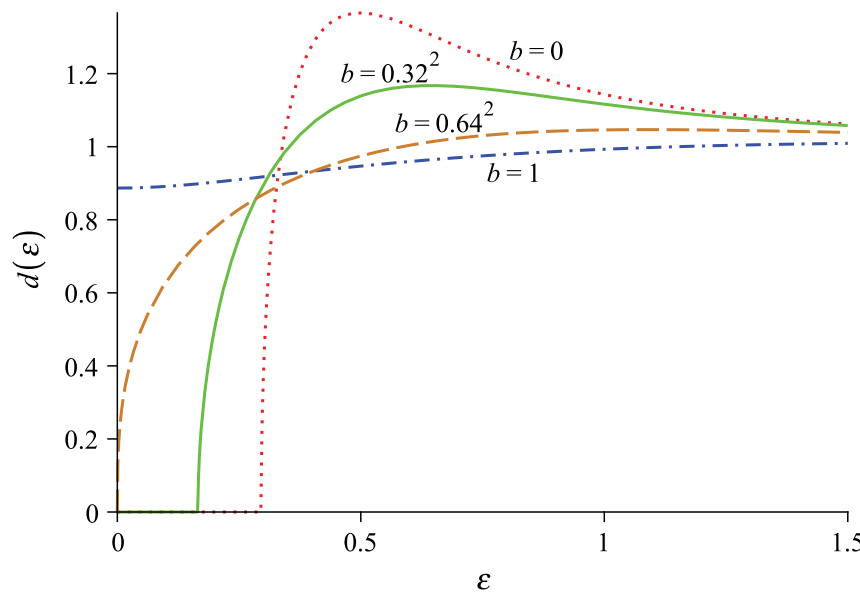

FIG. 11. (Color online) Magnetic-field dependence of the density of states of a chaotic Andreev billiard with phase difference $\phi=5 \pi / 6$ for $b=0$ (dotted line), $b=0.1024$ (solid line), $b=0.4096$ (dashed line), and $b=1$ (dashed dotted line).

field corresponding to $b \approx 0.1024$ closes the gap. In particular, the critical magnetic field for which the gap closes is given by ${ }^{35}$

$$
b_{c}=\frac{2 \cos (\phi / 2)}{1+\cos (\phi / 2)} \text {. }
$$

For ever increasing magnetic field, the density of states approaches 1 and we can see that a higher phase difference causes a faster convergence to this limit. Some examples are plotted in Fig. 12, and there we see that for $b=1$ the curve for $\phi=21 \pi / 22$ is nearly constant.

\section{Unequal leads}

Removing the restriction that the leads have equal size, we return to a sixth-order polynomial for $g$ and $G$ when substituting (60) into (58) and then (59). Expanding $G$ as a power series in $r$ via $G=\sum r^{n-1} C(\epsilon, \phi, n)$ now gives three starting values for $C(\epsilon, \phi, 1)$, and we choose the one that coincides with the result from the semiclassical diagrams, namely $\beta \beta^{*} /(1-\mathrm{i} \epsilon)$. Choosing the variable $y$ to represent the relative difference in the lead sizes

$$
y=\frac{N_{1}-N_{2}}{N}, \quad \beta=\cos \left(\frac{\phi}{2}\right)+\mathrm{i} y \sin \left(\frac{\phi}{2}\right),
$$

leads to a particularly compact solution, and as before, we can go through our roundabout route of finding the generating function of interest $H(\epsilon, \phi, y, r)$, which is recorded as (A3) in the appendix. Although it also was not possible to verify (other than at a large number of parameter values) this sixth-order equation, from it we can obtain the polynomial satisfied by $W(\epsilon, \phi, y)$ :

$$
\begin{aligned}
& {\left[\epsilon^{2} \hat{\beta}^{2} W^{4}+4 \epsilon \hat{\beta}^{2} W^{3}+\left(4 \hat{\beta}^{2}-\epsilon^{2}+2 \epsilon^{2} \hat{\beta}^{2}\right) W^{2}\right.} \\
& \left.\quad+4 \epsilon \hat{\beta}^{2} W-\epsilon^{2}+\epsilon^{2} \hat{\beta}^{2}\right](2+\epsilon W)^{2}+4 \epsilon^{2} y^{2}\left(1-\hat{\beta}^{2}\right)=0,
\end{aligned}
$$

where we have defined $\hat{\beta}=\cos (\phi / 2)$ as the real part of $\beta$ (which is equal to $\beta$ when the leads have equal size) and the evenness in $y$ follows from the symmetry under swapping the 

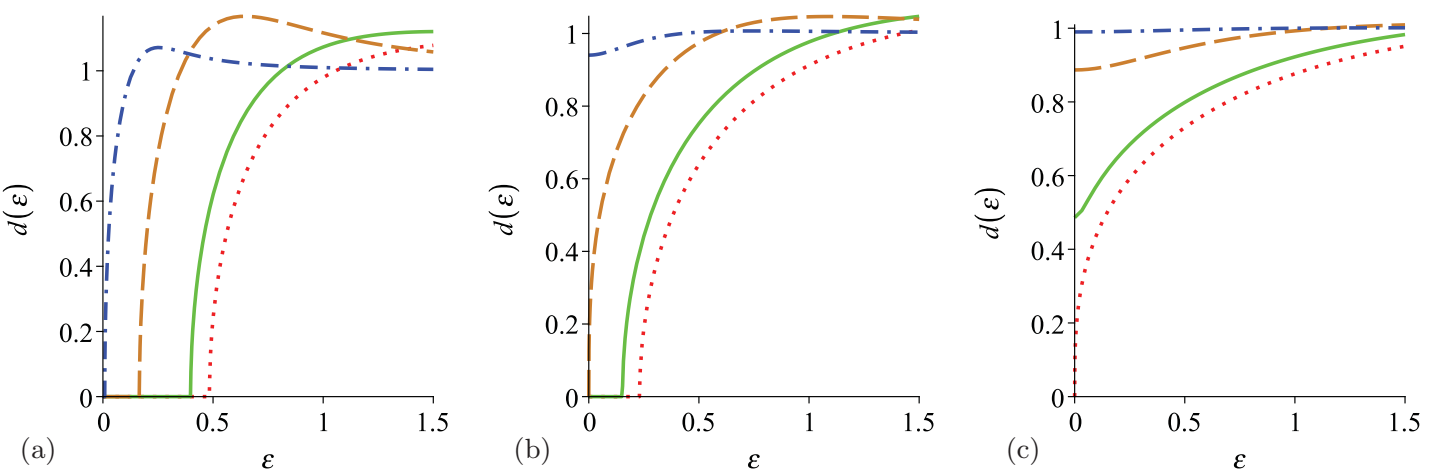

FIG. 12. (Color online) Phase dependence of the density of states of a chaotic Andreev billiard with phase difference $\phi=0$ (dotted line), $\phi=\pi / 2$ (solid line), $\phi=5 \pi / 6$ (dashed line), and $\phi=21 \pi / 22$ (dashed dotted line). (a) At magnetic field $b=0.1024,(\mathrm{~b})$ at $b=0.4096$, and (c) at $b=1$.

leads and $\phi$ to $-\phi$. The term in the square brackets is simply (64), and so we recover the result with equal leads when $y=0$. Likewise we can check that when we only have a single lead ( $y= \pm 1)$, we recover a factor corresponding to (12) so that the phase, as expected, no longer plays a role. From this equation, we can plot the density of states as in Fig. 13 and see how the difference in lead sizes $y$ interpolates between the result with equal leads above and the density of states with a single lead in (42). Note in particular that the peak in the density of states as the phase difference nears $\pi$ vanishes slowly as $y$ approaches \pm 1 , so that we can see a second gap appear in the density of states for leads differing distinctly in channel numbers [for example, see the solid line in Figs. 13(b) and 13(c)]. Numerically we can extract the critical value of $y$ for each phase difference $\phi$ above which we see a second gap. We plot this in Fig. 14, where we see that the second gap only appears for particularly unequal leads and at reasonable phase differences.

\section{EHRENFEST TIME DEPENDENCE}

So far we have been looking at the regime where the Ehrenfest time $\tau_{\mathrm{E}} \sim|\ln \hbar|$, the time below which wave packets propagate essentially classically (and above which wave interference dominates), is small compared to the dwell time $\tau_{\mathrm{d}}$, the typical time the trajectories spend inside the scattering region. This is the same limit described by RMT, and we have seen the agreement between semiclassics and RMT in Secs. IV and V above. Moving away from this limit, we can treat the typical effect of the Ehrenfest time on the correlation functions $C(\epsilon, n)$, for now for the simplest case of a single lead and no magnetic field. To contribute in the semiclassical limit, the correlated trajectories should have an action difference of the order of $\hbar$, which in turn means that the encounters have a duration of the order of the Ehrenfest time. Increasing this relative to the dwell time, or increasing the ratio $\tau=\tau_{\mathrm{E}} / \tau_{\mathrm{d}}$, then increases the possibility that all the trajectories travel together for their whole length in a correlated band. Likewise the probability of forming the diagrams (as in Fig. 4) considered before reduces. All told, the Ehrenfest time dependence $^{49}$ leads to the simple replacement

$$
C(\epsilon, \tau, n)=C(\epsilon, n) \mathrm{e}^{-(1-\mathrm{i} n \epsilon) \tau}+\frac{1-\mathrm{e}^{-(1-\mathrm{i} n \epsilon) \tau}}{1-\mathrm{i} n \epsilon} .
$$

This replacement leaves the $n=1$ term unchanged and had previously been shown for $n=2$ in Ref. 60 and $n=3$ in Ref. 39. The exponential growth of differences between trajectories due to the chaotic motion means that we just add the first term from the previous diagrams with encounters in (70) to the second term from the bands as their opposing length restrictions lead to a negligible overlap. In fact, this separation into two terms was shown ${ }^{73,74}$ to be a direct consequence of
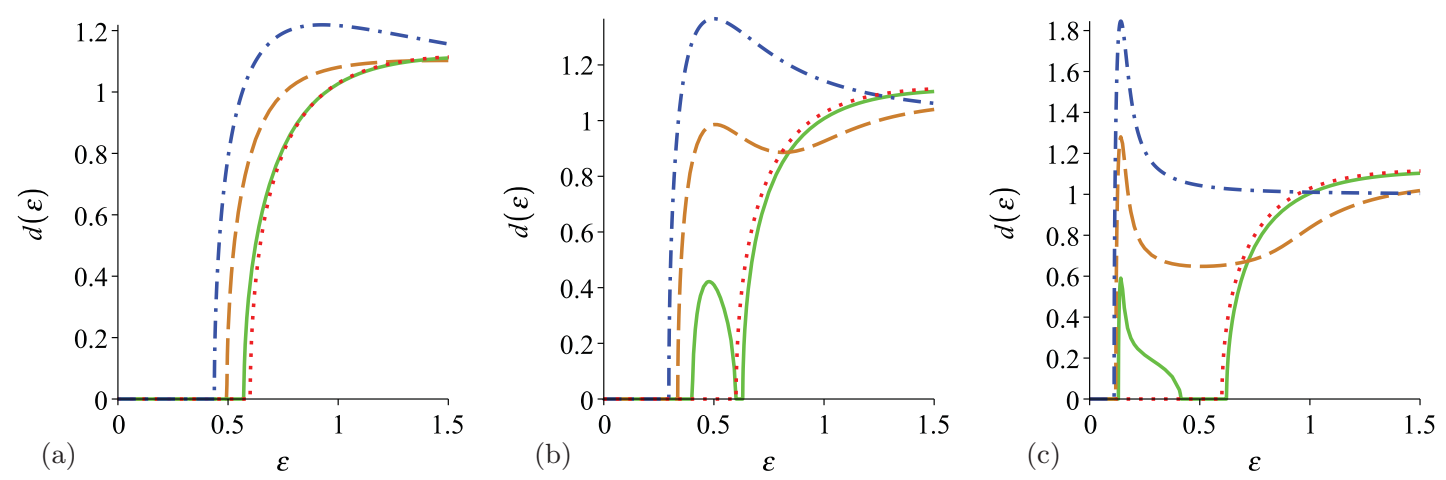

FIG. 13. (Color online) Dependence of the density of states of an Andreev billiard on the difference $y=\left(N_{1}-N_{2}\right) / N$ in size of the leads with $y=0$ (dashed dotted line), $y=4 / 5$ (dashed line), $y=\sqrt{24} / 5$, (solid line) and $y=1$ (dotted line). (a) At phase difference $\phi=2 \pi / 3$, (b) at $\phi=5 \pi / 6$, and (c) at $\phi=21 \pi / 22$. 


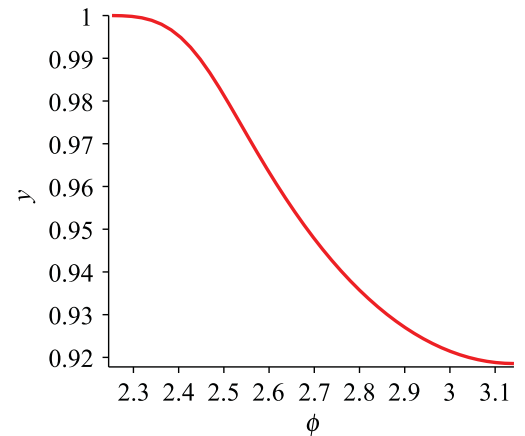

FIG. 14. (Color online) Critical value of the difference in the lead sizes $y$ as a function of the phase difference $\phi$ between the two leads above which a second gap appears in the density of states.

the splitting of the classical phase space into two virtually independent subsystems.

We leave the technical demonstration of (70) to Ref. 49, but the result follows by treating the diagrams considered before, which are created by sliding encounters together or into the lead (like the process depicted in Figs. 4 and 5), as part of a continuous deformation of a single diagram. With a suitable partition of this family, one can see that each set has the same $\tau_{\mathrm{E}}$ dependence and hence that (70) holds for all $n$. It is clear that in the limit $\tau=0$, (70) reduces to the previous (and hence RMT) results, while in the opposite limit, $\tau=\infty$, substituting (70) into (32) and performing a Poisson summation, we obtain the Bohr-Sommerfeld ${ }^{29}$ result

$$
d_{\mathrm{BS}}(\epsilon)=\left(\frac{\pi}{\epsilon}\right)^{2} \frac{\cosh (\pi / \epsilon)}{\sinh ^{2}(\pi / \epsilon)} .
$$

This result was previously found semiclassically by Ref. 30 and corresponds to the classical limit of bands of correlated trajectories.

For arbitrary Ehrenfest time dependence, we simply substitute the two terms in (70) into (32). With the second term we include $1-(1+\tau) \mathrm{e}^{-\tau}$ from the constant term (this turns out to simplify the expressions) and again perform a Poisson summation to obtain

$$
\begin{aligned}
d_{2}(\epsilon, \tau)= & -(1+\tau) \mathrm{e}^{-\tau}+2 \operatorname{Im} \sum_{n=1}^{\infty} \frac{(-1)^{n}}{n} \\
& \times \frac{\partial}{\partial \epsilon}\left(\frac{1-\mathrm{e}^{-(1-\mathrm{i} n \epsilon) \tau}}{1-\mathrm{i} n \epsilon}\right)=d_{\mathrm{BS}}(\epsilon)-\exp \left(-\frac{2 \pi k}{\epsilon}\right) \\
& \times\left(d_{\mathrm{BS}}(\epsilon)+\frac{2 k(\pi / \epsilon)^{2}}{\sinh (\pi / \epsilon)}\right),
\end{aligned}
$$

where $k=\lfloor(\epsilon \tau+\pi) /(2 \pi)\rfloor$ involves the floor function, and we see that this function is zero for $\epsilon \tau<\pi$.

Of course the first term in (70) also contributes, and when we substitute into (32) we obtain two further terms from the energy differential. These, however, may be written, using our semiclassical generating functions, as

$$
\begin{aligned}
d_{1}(\epsilon, \tau)= & \mathrm{e}^{-\tau}\left[1-2 \operatorname{Re} \mathrm{e}^{\mathrm{i} \epsilon \tau} H\left(\epsilon,-\mathrm{e}^{\mathrm{i} \epsilon \tau}\right)\right] \\
& +\tau \mathrm{e}^{-\tau}\left[1-2 \operatorname{Re} \mathrm{e}^{\mathrm{i} \epsilon \tau} G\left(\epsilon,-\mathrm{e}^{\mathrm{i} \epsilon \tau}\right)\right] .
\end{aligned}
$$
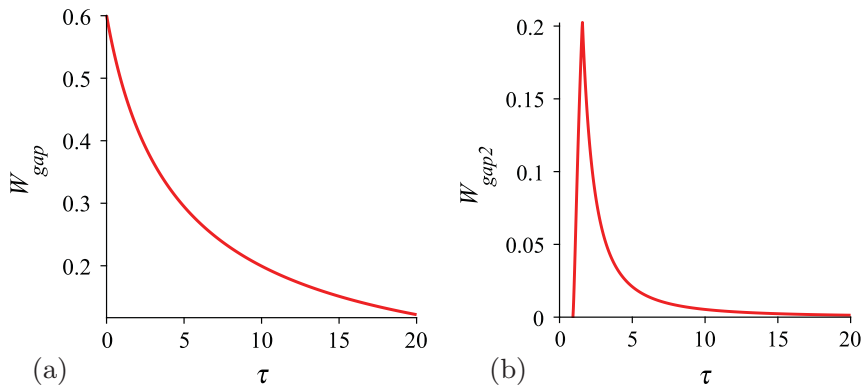

FIG. 15. (Color online) (a) Width (and end point) of the first gap and (b) width of the second gap as a function of $\tau$.

Because $G$ and $H$ are given by cubic equations, we can write this result explicitly as

$$
\begin{aligned}
d_{1}(\epsilon, \tau)= & \frac{\sqrt{3} \mathrm{e}^{-\tau}}{6 \epsilon} \operatorname{Re}\left[Q_{+}(\epsilon, \tau)-Q_{-}(\epsilon, \tau)\right] \\
& +\frac{\sqrt{3} \tau \mathrm{e}^{-\tau}}{6} \operatorname{Re}\left[P_{+}(\epsilon, \tau)-P_{-}(\epsilon, \tau)\right],
\end{aligned}
$$

where

$$
\begin{aligned}
Q_{ \pm}(\epsilon, \tau)= & {\left[8-\frac{24 \epsilon[1-\cos (\epsilon \tau)]}{\sin (\epsilon \tau)}-24 \epsilon^{2}\right.} \\
& -\frac{24 \epsilon^{2}[1-\cos (\epsilon \tau)]}{\sin ^{2}(\epsilon \tau)}+\frac{6 \epsilon^{3}[1-\cos (\epsilon \tau)]}{\sin (\epsilon \tau)} \\
& +\frac{2 \epsilon^{3}\left[2-3 \cos (\epsilon \tau)+\cos ^{3}(\epsilon \tau)\right]}{\sin ^{3}(\epsilon \tau)} \\
& \left. \pm \frac{6 \epsilon \sqrt{3 D}[1-\cos (\epsilon \tau)]}{\sin ^{2}(\epsilon \tau)}\right]^{\frac{1}{3}} \\
P_{ \pm}(\epsilon, \tau)= & {\left[\frac{36 \epsilon}{[1+\cos (\epsilon \tau)]^{2}}-\frac{9 \epsilon^{2} \sin (\epsilon \tau)}{[1+\cos (\epsilon \tau)]^{3}}\right.} \\
& \left.+\frac{\epsilon^{3}}{[1+\cos (\epsilon \tau)]^{3}} \pm \frac{3 \sqrt{3 D}}{[1+\cos (\epsilon \tau)]^{2}}\right]^{\frac{1}{3}}
\end{aligned}
$$

These all involve the same discriminant $D$ and so the differences in (74) are only real [and hence $d_{1}(\epsilon, \tau)$ itself is nonzero] when

$$
\begin{aligned}
D(\epsilon, \tau)= & \epsilon^{4}-8 \epsilon^{3} \sin (\epsilon \tau)+4 \epsilon^{2}[5+6 \cos (\epsilon \tau)] \\
& +24 \epsilon \sin (\epsilon \tau)-8[1+\cos (\epsilon \tau)]
\end{aligned}
$$

is positive. Recalling that the second contribution is zero up to $\epsilon \tau=\pi$, the complete density of states is therefore zero up to the first root of $D(\epsilon, \tau)$. The width of this gap is then solely determined by the contribution from quantum-interference terms given by the trajectories with encounters. The hard gap up to the first root shrinks as $\tau$ increases [see Fig. 15(a)], and when taking the limit $\tau \rightarrow \infty$ while keeping the product $\epsilon \tau$ constant, (77) reduces to $-8[1+\cos (\epsilon \tau)]$, which has its first root at $\epsilon \tau=\pi$. The gap then approaches $E=\pi E_{\mathrm{E}}$ for $\tau \gg 1$, where $E_{\mathrm{E}}=\hbar / 2 \tau_{\mathrm{E}}$ is the Ehrenfest energy. So one indeed observes a hard gap up to $\pi E_{\mathrm{E}}$ in the limit $\tau \rightarrow \infty$ at fixed $\epsilon \tau$, in agreement with the quasiclassical result of Ref. 40 .

Alongside this reduction in size of the first gap, which was predicted by effective RMT, ${ }^{13}$ when $\tau \geqslant 0.916$ the discriminant (77) has additional roots. Between the second and 

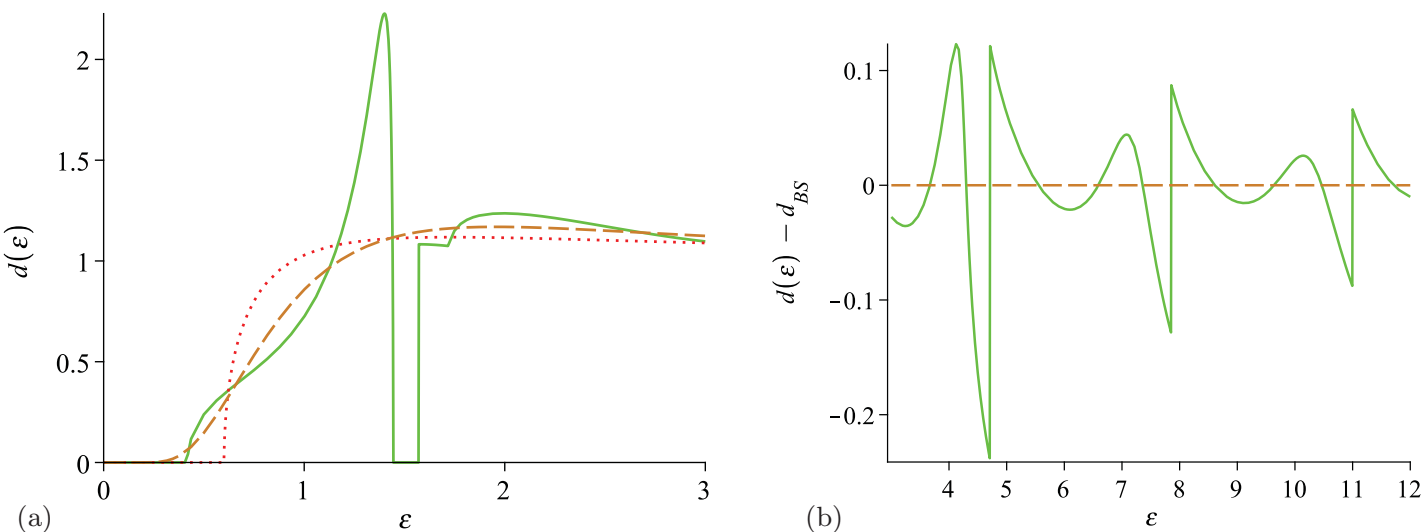

FIG. 16. (Color online) (a) Density of states for $\tau=\tau_{\mathrm{E}} / \tau_{\mathrm{d}}=2$ (solid line), along with the BS (dashed) limit $\tau \rightarrow \infty$ and the RMT (dotted) limit $\tau=0$, showing a second gap just below $\epsilon \tau=\pi$. (b) Ehrenfest time related $2 \pi / \tau$-periodic oscillations in the density of states after subtracting the BS curve.

third root, $D(\epsilon, \tau)$ is also negative and a second gap appears. As $\tau$ increases, the roots spread apart so the gap widens. For example, the complete density of states for $\tau=2$ is shown in Fig. 16(a) along with the oscillatory behavior visible at larger energies (with period $2 \pi / \tau$ ) in Fig. 16(b). There the second gap is clearly visible and only ends when the second contribution $d_{2}(\epsilon, \tau)$ becomes nonzero at $\epsilon \tau=\pi$. In fact, for $\tau>\pi / 2$ the third root of $D(\epsilon, \tau)$ is beyond $\epsilon \tau=\pi$, so the second gap is cut short by the jump in the contribution $d_{2}(\epsilon, \tau)$. Since the second root also increases with increasing $\tau$, the gap shrinks again, as can be seen in Fig. 15(b).

To illustrate this behavior further, the density of states is shown for different values of $\tau$ in Fig. 17. One can see first the formation and then the shrinking of the second gap. As can be seen in the inset of Fig. 17(b), the second gap persists even for large values of $\tau$ and the size of the first hard gap converges slowly to $\epsilon \tau=\pi$. The plot for $\tau=20$ also shows how the density of states converges to the BS result.

\section{A. Effective RMT}

As mentioned above, the shrinking of the first gap has been predicted by effective RMT where the effect of the
Ehrenfest time is mimicked ${ }^{37}$ by reducing the number of channels in the lead by a factor $\mathrm{e}^{\tau}$ (to correspond to the part of classical phase space where the trajectories are longer than the Ehrenfest time) and to multiply the scattering matrix by the phase $\mathrm{e}^{\mathrm{i} \epsilon \tau / 2}$ to represent the energy dependence of the lead. The random-matrix diagrammatic averaging leads to the set of equations $^{13,44}$

$$
\begin{aligned}
W^{2}+1 & =W_{2}^{2} \\
W+W_{2} \sin u & =-\frac{\epsilon}{2} W_{2}\left(W_{2}+\cos u+W \sin u\right),
\end{aligned}
$$

where $u=\epsilon \tau / 2$ and the density of states is given by (for $u<\pi / 2)$

$$
d(\epsilon, \tau)=-\mathrm{e}^{-\tau} \operatorname{Im}\left(W-\frac{u}{\cos u} W_{2}\right) .
$$

The equations in (78) can be simplified to obtain a cubic for $W$ (and $W_{2}$ ), and in this form we can compare with our semiclassical results. In fact, making the substitution $H=[\mathrm{i} W-1] / 2 r$ and setting $r=-\exp (\mathrm{i} \in \tau)$ to get the first part in (73) in the form of the first term in (79), we obtain exactly the same polynomial and hence agreement. Likewise when we substitute $G=-\left[i u W_{2} / \cos u+\tau\right] / 2 r \tau$,
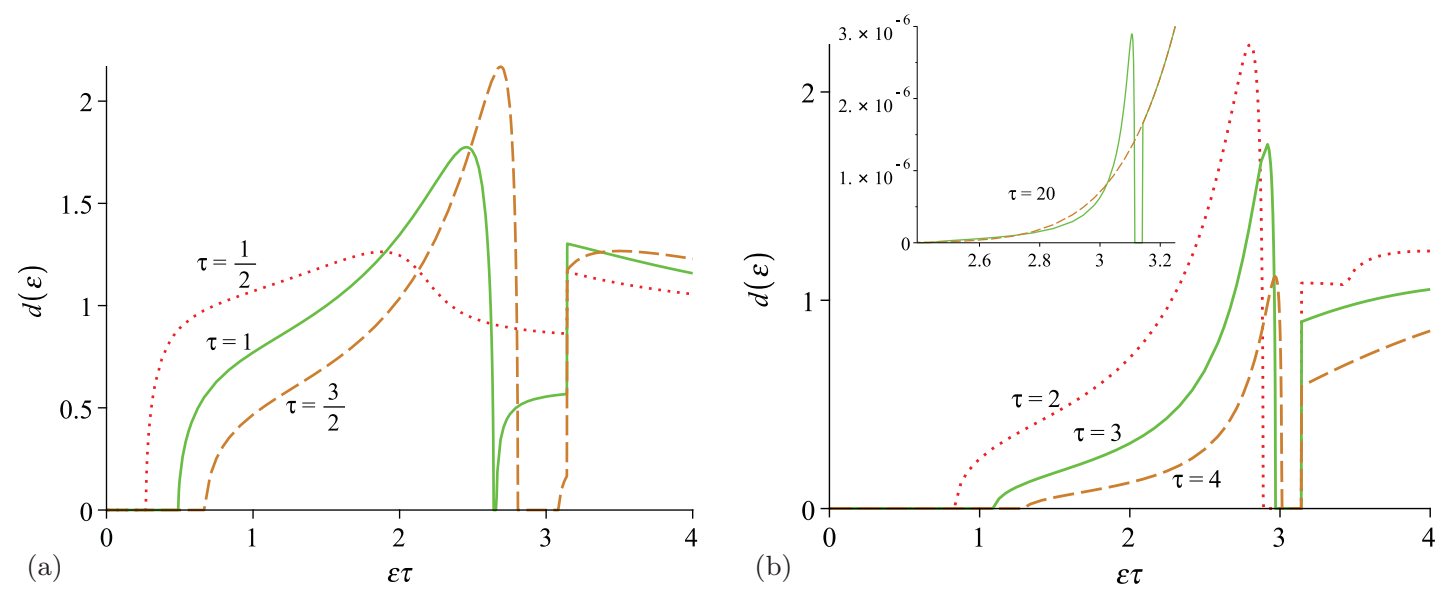

FIG. 17. (Color online) Density of states as a function of $\epsilon \tau=E / E_{\mathrm{E}}$ for various values of $\tau$ showing the appearance of a second gap below $\epsilon \tau=\pi$. Inset: Density of states for $\tau=20$ (solid line) together with the BS limit (dashed). 
we obtain the same polynomial for the second part, albeit with the real offset $\tan u$, which does not affect the density of states.

Of course this agreement provides semiclassical support for the phenomenological approach of effective RMT as well as showing that (79) is valid for $u$ beyond $\pi / 2$. In principle then the second gap could also have been found using effective RMT, but of course effective RMT misses the second contribution to the density of states $d_{2}(\epsilon, \tau)$. This contribution turns out to be straightforward to obtain semiclassically, and can be compared to the bands treated in Ref. 40 .

\section{B. Two superconducting leads}

If we include the effect of a symmetry-breaking magnetic field, then, because of the way this affects the contribution of different sized encounters (as described in Sec. IVD), such a simple replacement as in (70) no longer holds. This situation has, however, been treated using effective RMT, ${ }^{44}$ allowing them to also determine how the critical magnetic field (at which the gap closes) depends on the Ehrenfest time. Without the simple replacement of the magnetic-fielddependent correlations functions, we are currently unable to confirm this result semiclassically. But if we look at two superconducting leads (for simplicity of equal size) at different phase, this effect can be included in the channel sum and treated as above (the effective RMT result can be found by a simple modification of the treatment in Ref. 44). Important to remember is that the second part [of (70)] corresponds to bands of trajectories that are correlated for their whole length, and so they all start and end together (in the same leads). Therefore, the second contribution has to be multiplied by $[1+\cos (n \phi)] / 2$, leading to

$$
\begin{aligned}
C(\epsilon, \phi, \tau, n)= & C(\epsilon, \phi, n) \mathrm{e}^{-(1-\mathrm{i} n \epsilon) \tau} \\
& +\frac{1+\cos (n \phi)}{2} \frac{1-\mathrm{e}^{-(1-\mathrm{i} n \epsilon) \tau}}{1-\mathrm{i} n \epsilon} .
\end{aligned}
$$

The first part of the density of states for nonzero Ehrenfest time then remains as in (73) but with $G(\epsilon, r)$ and $H(\epsilon, r)$ replaced by $G(\epsilon, \phi, r)$ and $H(\epsilon, \phi, r)$, respectively. The second contribution in this case, however, may be written as the average of the $\phi=0$ contribution and a contribution with the full phase difference $\phi$,

$$
d_{2}(\epsilon, \phi, \tau)=\frac{1}{2}\left[d_{2}^{\prime}(\epsilon, 0, \tau)+d_{2}^{\prime}(\epsilon, \phi, \tau)\right] .
$$

Here $d_{2}^{\prime}(\epsilon, \phi, \tau)$ may be again written as the sum of the $\tau=\infty$ result,

$$
\begin{aligned}
d_{2}^{\prime(1)}(\epsilon, \phi, \tau)= & \frac{\pi}{2 \epsilon^{2} \sinh ^{2}(\pi / \epsilon)}\left[\left(\pi+2 \pi k_{1}-\phi\right)\right. \\
& \times \cosh \left(\frac{\pi-2 \pi k_{1}+\phi}{\epsilon}\right)+\left(\pi-2 \pi k_{1}+\phi\right) \\
& \left.\times \cosh \left(\frac{\pi+2 \pi k_{1}-\phi}{\epsilon}\right)\right],
\end{aligned}
$$

and some correction

$$
\begin{aligned}
d_{2}^{\prime(2)}(\epsilon, \phi, \tau)= & -\frac{\pi}{2 \epsilon^{2} \sinh ^{2}(\pi / \epsilon)}\left\{\left[\pi \cosh \left(\frac{\pi}{\epsilon}\right)\right.\right. \\
& \left.+\left(2 \pi k_{2}-\phi\right) \sinh \left(\frac{\pi}{\epsilon}\right)\right] \mathrm{e}^{-\frac{2 \pi k_{2}-\phi}{\epsilon}} \\
& +\left[\pi \cosh \left(\frac{\pi}{\epsilon}\right)+\left(2 \pi k_{3}+\phi\right) \sinh \left(\frac{\pi}{\epsilon}\right)\right] \\
& \left.\times \mathrm{e}^{-\frac{2 \pi k_{3}+\phi}{\epsilon}}\right\},
\end{aligned}
$$

with $k_{1}=\lfloor(\pi+\phi) /(2 \pi)\rfloor, k_{2}=\lfloor(\epsilon \tau+\pi+\phi) /(2 \pi)\rfloor$, and $k_{3}=\lfloor(\epsilon \tau+\pi-\phi) /(2 \pi)\rfloor$. Since the $k_{i}$ and $\phi$ only occur in the combinations $2 \pi k_{1}-\phi, 2 \pi k_{2}-\phi$, and $2 \pi k_{3}+\phi$, it is obvious that these contributions have oscillations in the phase $\phi$ with period $2 \pi$. It can also be easily seen that for $\phi=0$, the previous result for the density of states in the Ehrenfest regime is reproduced.

With $|\phi|<\pi$ we have $k_{1}=k_{2}=k_{3}=0$ for $\epsilon \tau<\pi-|\phi|$. Therefore, one again sees that $d_{2}=0$ as long as $\epsilon \tau<\pi-|\phi|$. The first part $d_{2}^{\prime(1)}$ equals the Bohr-Sommerfeld result (71), so in the limit $\tau=\infty$ this result is reproduced again. The oscillations in $\epsilon$ seen in the $\phi=0$ case which have a period of $2 \pi / \tau$ can still be seen due to the fact that the $\phi=0$ result enters $d_{2}(\epsilon, \phi, \tau)$ even if $\phi \neq 0$. However, one gets additional (but smaller) steps at energies satisfying $\epsilon=[(2 m-1) \pi \mp \phi] / \tau$ for integer $m$.
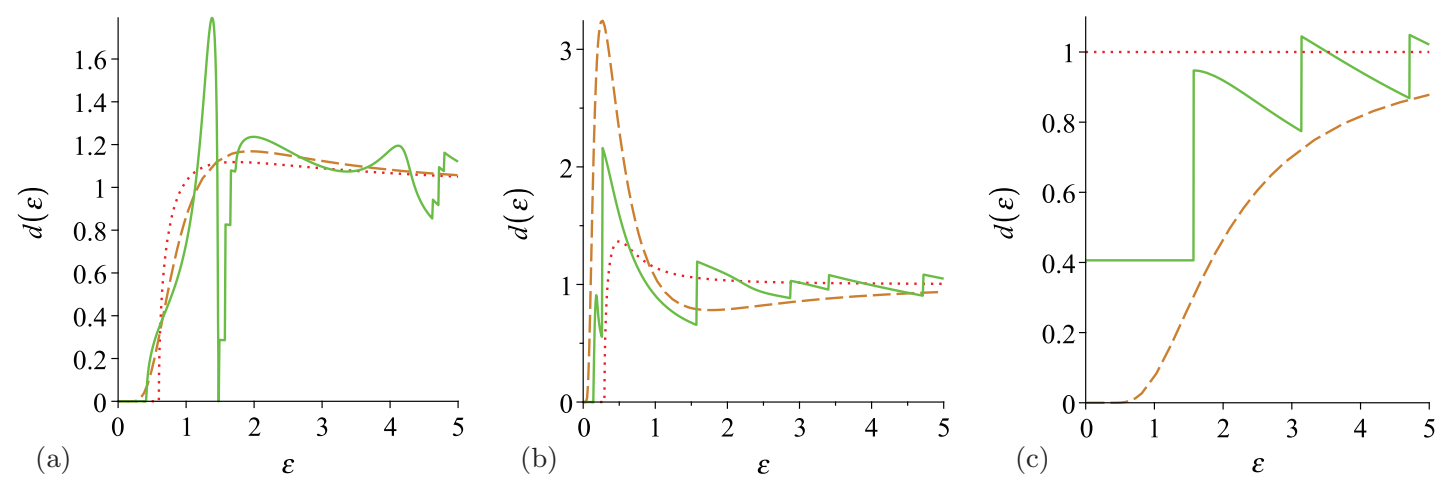

FIG. 18. (Color online) Density of states for $\tau=2$ (solid line) along with the $\tau=0$ (dotted) and $\tau=\infty$ (dashed) limits for a chaotic Andreev billiard with phase difference (a) $\phi=\pi / 18$, (b) $\phi=5 \pi / 6$, and (c) $\phi=\pi$. 

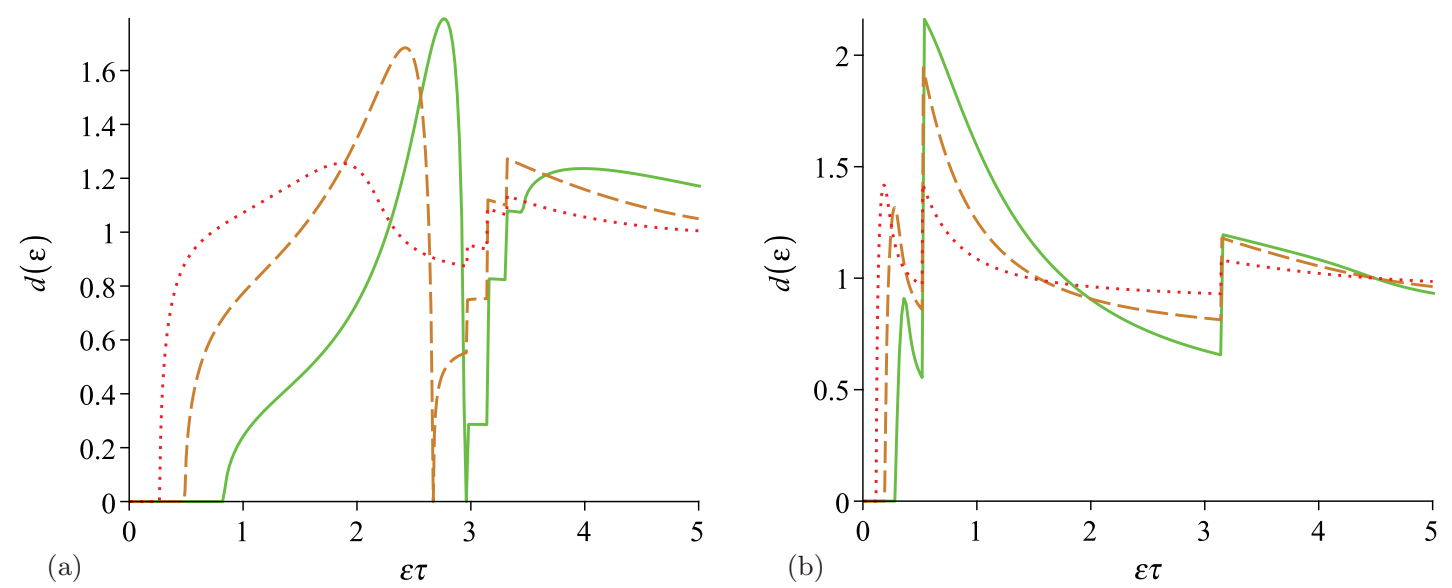

FIG. 19. (Color online) Density of states for $\tau=1 / 2$ (dotted line), $\tau=1$ (dashed), and $\tau=2$ (solid) showing the phase-dependent jumps for phase difference (a) $\phi=\pi / 18$ and (b) $\phi=5 \pi / 6$.

We plot the density of states for $\tau=2$, along with the $\tau=0$ and $\tau=\infty$ limits, in Fig. 18 for different values of the phase difference. We can see that as the phase difference increases, the second intermediate gap [cf. Fig. 16(a)] shrinks quickly. The reason for this shrinking is twofold: On the one hand, the gap in the RMT-like contribution shrinks, and on the other hand, the second contribution is zero only up to $\epsilon \tau=\pi-|\phi|$. Moreover, if $\phi \rightarrow \pi$, the modified correlation function tends to zero so the density of states converges to $(1+\tau) \mathrm{e}^{-\tau}+d_{2}(\epsilon, \tau)$. For a finer look at the Ehrenfest time dependence and the phase-dependent jumps, we plot the density of states for $\tau=$ $1 / 2,1$, and 2 for phases $\phi=\pi / 18$ and $5 \pi / 6$ in Fig. 19.

\section{CONCLUSIONS}

From the semiclassical treatment of the density of states of chaotic Andreev billiards, we have seen how fine correlations between ever larger sets of classical trajectories lead to the interference effects, which cause a hard gap in the density of states. This treatment (cf. the reservations in Ref. 38) builds on the recent advances in identifying, ${ }^{55}$ codifying, ${ }^{56,57}$ and generating ${ }^{47}$ the semiclassical contributions, and, because of the slow convergence of the expansion for the density of states in (15), relies on the ability to treat correlations between $n$ trajectories for essentially all $n$. The correlations between these trajectories, encoded in encounter regions where they differ slightly, are represented by simple (tree) diagrams. These diagrams are related to those that appear for the conductance, ${ }^{56}$ say where for increasing $n$ they cause ever decreasing (in inverse channel number) corrections; here, though, they all contribute with roughly the same (slowly decreasing) importance. It is because we need to treat all orders that Andreev billiards are so interesting and the resultant effects so large.

Along with obtaining the minigap, found by RMT, ${ }^{27}$ for a billiard with a single lead, we could also obtain the full result for the density of states of an Andreev billiard with two superconducting leads at phase difference $\phi$, treated using RMT in Ref. 35. The semiclassical paths that connect the two leads accumulate phases $\mathrm{e}^{ \pm \mathrm{i} \phi}$ and cause the gap to shrink with increasing phase difference. It was also possible to treat the effect of a time-reversal symmetry-breaking magnetic field $b$, considered with RMT in Ref. 35, which makes the formation of the classical trajectory sets, traversed in opposite directions by an electron and a hole, less likely. This in turn leads to a reduction of the minigap and a smoothing of the density of states, especially for large phase differences $\phi$. We have found that in the limits $\phi \rightarrow \pi$ and $b \rightarrow \infty$, quantum effects vanish and the density of states becomes identical to the density of states of the isolated billiard.

The agreement shown here between the semiclassical and the RMT results may lead one to wonder about the deeper connections between the two methods. Indeed, the diagrammatic methods ${ }^{69}$ used in RMT, which also use recursion relations over planar diagrams, bear some resemblance to the tree recursions here, but unfortunately any correspondence between the two is somewhat obscured by the transformation from the generating function $G$ to the generating function $H$. It is also worth pointing out that our semiclassical treatment (with its inherent semiclassical limits) gives us access to the typical and universal density of states of chaotic systems. However, there can be further effects that change the shape of the density of states, for example scarring, ${ }^{75}$ classical Lyapunov exponent fluctuations, ${ }^{76}$ and disorder, ${ }^{77}$

Of course all our results in this paper (and the RMT one ${ }^{27,35}$ ) are only valid to leading order in inverse channel number. With the formalism shown in this paper, to go to subleading order we only require a way of generating the possible semiclassical diagrams. The contribution of each ${ }^{56,57}$ and how they affect the density of states is known in principle, but the key problem is that the structure we used here breaks down, namely that in the tree recursions when we cut a rooted plane tree at a node, we created additional rooted plane trees. ${ }^{47}$ How to treat the possible diagrams, which include closed loops, etc., though generated for $n=1$ in Ref. 56 and $n=2$ in Ref. 57, by cutting open closed periodic orbits, remains unclear. However, the treatment for $n=1$ and 2 makes it clear that the diagrams that contribute at order $\left(1 / N^{m}, n\right)$ are related to those that contribute at order $\left(1 / N^{m-1}, n+1\right)$, raising the possibility of a recursive treatment starting from the leading-order diagrams described here. 
Worth noting is that the semiclassical techniques we used here are only valid up to the Heisenberg time, meaning that we have no access to the density of states on energy scales of the order of the mean level spacing. Though for ballistic transport, the Heisenberg time is much longer than the average dwell time (so the mean level spacing is much smaller than the Thouless energy), importantly the RMT treatment ${ }^{78}$ shows that a microscopic gap persists in this regime even when the time-reversal symmetry is completely broken (by the magnetic field, say). It may be possible that applying the semiclassical treatment of times longer than the Heisenberg time for closed systems ${ }^{79,80}$ to transport would allow one to access this regime as well.

In the opposite regime, however, that of the Ehrenfest time, semiclassics provides a surprisingly simple result ${ }^{49}$ allowing complete access to the crossover from the universal RMT regime to the more classical Bohr-Sommerfeld regime. The gap shrinks due to the suppression of the formation of encounters, while a new class of diagrams (correlated bands) becomes possible. Interestingly, the contribution from trajectories with encounters agrees exactly with the results from effective RMT, ${ }^{13}$ so our semiclassical result provides support for this phenomenological approach. Of course, effective RMT misses the bands of correlated trajectories (cf. those in Ref. 40), which, combined with the other contribution, lead to new effects, most notably a second gap in the density of states for intermediate Ehrenfest times.

\section{ACKNOWLEDGMENTS}

The authors would like to thank I. Adagideli for useful conversations, and we gratefully acknowledge the Deutsche Forschungsgemeinschaft within GRK 638 (D.W., K.R.) and FOR 760 (K.R.), the National Science Foundation under Grant No. 0604859 (G.B.), CEA Eurotalent (C.P.), and the Alexander von Humboldt Foundation (J.K., C.P.) for funding.

\section{APPENDIX: FURTHER GENERATING FUNCTIONS}

The intermediate generating function $I(\epsilon, r)$ for the billiard with a single lead and no magnetic field in Sec. IV B is given by

$$
\begin{aligned}
1- & {\left[(1-a)^{2}+6 r+(1+a)^{2} r^{2}\right] I+\left[4(1-a)^{3}-\left(8+20 a^{2}-a^{4}\right) r+4(1+a)^{3} r^{2}\right] r I^{2} } \\
& +\left[4(1-a)^{3}-\left(16-24 a+44 a^{2}-8 a^{3}-a^{4}\right) r+2\left(12+32 a^{2}-a^{4}\right) r^{2}\right. \\
& \left.-\left(16+24 a+44 a^{2}+8 a^{3}-a^{4}\right) r^{3}+4(1+a)^{3} r^{4}\right] r I^{3}=0,
\end{aligned}
$$

where we set $a=\mathrm{i} \epsilon$.

The generating function $H(\epsilon, \phi, b, r)$ for the billiard with equal leads at phase difference $\phi$ and magnetic field $b$ in Sec. V B is given by

$$
\begin{aligned}
\beta^{2} & -\left[(1-a+b)^{2}+r^{2}-2 r(1-a+b)\left(2 \beta^{2}-1\right)\right] H-r\left\{(1-a+b)(1-3 a+7 b)-2 r\left[1+5 b+b^{2}\right.\right. \\
& \left.\left.-(3+2 b) a+a^{2}\right]\left(2 \beta^{2}-1\right)+r^{2}(1-2 a+2 b)\right\} H^{2}+r^{2}\left\{-b(19 b+10)+2 a(9 b+1)-3 a^{2}+2 r[2 b(3 b+4)\right. \\
& \left.\left.-2 a(4 b+1)+2 a^{2}\right]\left(2 \beta^{2}-1\right)+r^{2}\left[-b(b+6)+2 a(b+1)-a^{2}\right]\right\} H^{3}-r^{3}\left\{b(25 b+4)-14 a b+a^{2}-2 r[b(13 b+4)\right. \\
& \left.\left.-10 a b+a^{2}\right]\left(2 \beta^{2}-1\right)+r^{2}\left[b(5 b+4)-6 a b+a^{2}\right]\right\} H^{4}-4 r^{4} b\left[4 b-a-2 r(3 b-a)\left(2 \beta^{2}-1\right)+r^{2}(2 b-a)\right] H^{5} \\
& -4 r^{5} b^{2}\left[1+r^{2}-2 r\left(2 \beta^{2}-1\right)\right] H^{6}=0,
\end{aligned}
$$

where we also used $a=\mathrm{i} \epsilon$. For the billiard with unequal leads and no magnetic field in Sec. VC, the generating function $H(\epsilon, \phi, y, r)$ is given by

$$
\begin{aligned}
\beta \beta^{*} & (1-a)^{2}+\beta \beta^{*} r^{2}-\left(\beta^{2}+\beta^{* 2}\right)(1-a) r+\left(-(1-a)^{4}+r\left\{\left(\beta+\beta^{*}\right)^{2}\left(1-a^{3}\right)+\left[3\left(\beta+\beta^{*}\right)^{2}+2 \beta \beta^{*}\right] a(a-1)\right\}\right. \\
+ & \left.r^{2}\left\{\left[3\left(\beta+\beta^{*}\right)^{2}-2 \beta \beta^{*}-2\right] a(2-a)+2\left(1+\beta+\beta^{*}\right)\left(1-\beta-\beta^{*}\right)\right\}+r^{3}\left\{\left(\beta+\beta^{*}\right)^{2}-a\left[\left(\beta+\beta^{*}\right)^{2}+2 \beta \beta^{*}\right]\right\}-r^{4}\right) H \\
+ & r\left\{(1-a)^{3}(5 a-1)+\left[\left(\beta+\beta^{*}\right)^{2}\left(1-7 a-7 a^{3}+a^{4}\right)+\left(3 \beta+4 \beta^{*}\right)\left(4 \beta+3 \beta^{*}\right) a^{2}\right] r+2\left(1+\beta+\beta^{*}\right)\left(1-\beta-\beta^{*}\right)\right. \\
& \times\left(1-6 a-2 a^{3}\right) r^{2}-\left(15 \beta^{2}+15 \beta^{* 2}-14+28 \beta \beta^{*}\right) a^{2} r^{2}+\left[\left(\beta+\beta^{*}\right)^{2}(1-5 a)+\left(3 \beta^{2}+3 \beta^{* 2}+7 \beta \beta^{*}\right) a^{2}\right] r^{3} \\
+ & \left.(4 a-1) r^{4}\right\} H^{2}+a r^{2}\left[2(1-a)^{2}(2-5 a)+\left(\beta+\beta^{*}\right)^{2}\left(4 a^{3}-15 a^{2}+15 a-4\right) r+2\left(1+\beta+\beta^{*}\right)\left(1-\beta-\beta^{*}\right)\right. \\
& \left.\times\left(a^{3}-8 a^{2}+12 a-4\right) r^{2}+\left(\beta+\beta^{*}\right)^{2}\left(-3 a^{2}+9 a-4\right) r^{3}+(4-6 a) r^{4}\right\} H^{3}+a^{2} r^{3}\left[16 a-10 a^{2}-6+\left(\beta+\beta^{*}\right)^{2}\right. \\
& \left.\times\left(6-13 a+6 a^{2}\right) r+2\left(1+\beta+\beta^{*}\right)\left(1-\beta-\beta^{*}\right)\left(6-10 a+3 a^{2}\right) r^{2}+\left(\beta+\beta^{*}\right)^{2}\left(6-7 a+a^{2}\right) r^{3}+(4 a-6) r^{4}\right] H^{4} \\
+ & a^{3} r^{4}\left[4-5 a+4\left(\beta+\beta^{*}\right)^{2}(a-1) r+2\left(1+\beta+\beta^{*}\right)\left(1-\beta-\beta^{*}\right)(3 a-4) r^{2}+\left(\beta+\beta^{*}\right)^{2}(2 a-4) r^{3}+(4-a) r^{4}\right] H^{5} \\
+ & a^{4} r^{5}\left[-1-r^{4}+r\left(1+r^{2}\right)\left(\beta+\beta^{*}\right)^{2}+2 r^{2}\left(1+\beta+\beta^{*}\right)\left(1-\beta-\beta^{*}\right)\right] H^{6}=0,
\end{aligned}
$$

likewise with $a=\mathrm{i} \epsilon$. 
*jack.kuipers@physik.uni-regensburg.de

${ }^{\dagger}$ thomas.engl@physik.uni-regensburg.de

${ }^{1}$ S. Guéron, H. Pothier, N. O. Birge, D. Esteve, and M. H. Devoret, Phys. Rev. Lett. 77, 3025 (1996).

${ }^{2}$ A. F. Morpurgo, S. Holl, B. J. van Wees, T. M. Klapwijk, and G. Borghs, Phys. Rev. Lett. 78, 2636 (1997).

${ }^{3}$ S. G. den Hartog, B. J. van Wees, Yu. V. Nazarov, T. M. Klapwijk, and G. Borghs, Phys. Rev. Lett. 79, 3250 (1997).

${ }^{4}$ M. Jakob, H. Stahl, J. Knoch, J. Appenzeller, B. Lengeler, H. Hardtdegen, and H. Lüth, Appl. Phys. Lett. 76, 1152 (2000).

${ }^{5}$ N. Moussy, H. Courtois, and B. Pannetier, Europhys. Lett. 55, 861 (2001).

${ }^{6}$ M. Vinet, C. Chapelier, and F. Lefloch, Phys. Rev. B 63, 165420 (2001).

${ }^{7}$ J. Eroms, M. Tolkiehn, D. Weiss, U. Rössler, J. D. Boeck, and G. Borghs, Europhys. Lett. 58, 569 (2002).

${ }^{8}$ W. Escoffier, C. Chapelier, N. Hadacek, and J.-C. Villégier, Phys. Rev. Lett. 93, 217005 (2004).

${ }^{9}$ W. Escoffier, C. Chapelier, and F. Lefloch, Phys. Rev. B 72, 140502 (2005).

${ }^{10}$ C. J. Lambert and R. Raimondi, J. Phys.: Condens. Matter 10, 901 (1998).

${ }^{11}$ A. Altland, B. D. Simons, and D. Taras-Semchuk, Adv. Phys. 49, 321 (2000).

${ }^{12}$ D. Taras-Semchuk and A. Altland, Phys. Rev. B 64, 014512 (2001).

${ }^{13}$ C. W. J. Beenakker, Lect. Notes Phys. 667, 131 (2005).

${ }^{14}$ D. Saint-James, J. Phys. (France) 25, 899 (1964).

${ }^{15}$ A. F. Andreev, Zh. Eksp. Teor. Fiz. 46, 1823 (1964) [Sov. Phys. JETP 19, 1228 (1964)].

${ }^{16}$ W. L. McMillan, Phys. Rev. 175, 537 (1968).

${ }^{17}$ C. W. J. Beenakker, Rev. Mod. Phys. 69, 731 (1997).

${ }^{18}$ B. J. van Wees and H. Takayanagi, in Mesoscopic Electron Transport, edited by L. L. Sohn, L. P. Kouwenhoven, and G. Schön NATO Advanced Study Institute Series E: 345 (Kluwer, Dordrecht, 1997).

${ }^{19}$ V. A. Gopar, J. A. Méndez-Bermúdez, and A. H. Aly, Phys. Rev. B 79, 245412 (2009).

${ }^{20}$ R. S. Whitney and Ph. Jacquod, Phys. Rev. Lett. 103, 247002 (2009).

${ }^{21}$ M. C. Goorden, Ph. Jacquod, and J. Weiss, Phys. Rev. Lett. 100, 067001 (2008).

${ }^{22}$ P. Cadden-Zimansky, J. Wei, and V. Chandrasekhar, Nat. Phys. 5, 393 (2009).

${ }^{23} \mathrm{Ph}$. Jacquod and R. S. Whitney, Europhys. Lett. 91, 67009 (2010).

${ }^{24}$ B.-R. Choi, A. E. Hansen, T. Kontos, C. Hoffmann, S. Oberholzer, W. Belzig, C. Schönenberger, T. Akazaki, and H. Takayanagi, Phys. Rev. B 72, 024501 (2005).

${ }^{25}$ J. Eroms and D. Weiss, Appl. Phys. A 89, 639 (2007).

${ }^{26}$ I. Kosztin, D. L. Maslov, and P. M. Goldbart, Phys. Rev. Lett. 75, 1735 (1995).

${ }^{27}$ J. A. Melsen, P. W. Brouwer, K. M. Frahm, and C. W. J. Beenakker, Europhys. Lett. 35, 7 (1996).

${ }^{28}$ A. Lodder and Y. V. Nazarov, Phys. Rev. B 58, 5783 (1998).

${ }^{29}$ H. Schomerus and C. W. J. Beenakker, Phys. Rev. Lett. 82, 2951 (1999).

${ }^{30}$ W. Ihra, M. Leadbeater, J. L. Vega, and K. Richter, Eur. Phys. J. B 21, 425 (2001).

${ }^{31}$ W. Ihra and K. Richter, Physica E 9, 362 (2001).

${ }^{32}$ J. Cserti, A. Kormányos, Z. Kaufmann, J. Koltai, and C. J. Lambert, Phys. Rev. Lett. 89, 057001 (2002).
${ }^{33}$ I. Adagideli and P. M. Goldbart, Int. J. Mod. Phys. B 16, 1381 (2002).

${ }^{34}$ O. Zaitsev, J. Phys. A 39, L467 (2006).

${ }^{35}$ J. A. Melsen, P. W. Brouwer, K. M. Frahm, and C. W. J. Beenakker, Phys. Scr. T 69, 223 (1997).

${ }^{36}$ I. Adagideli and C. W. J. Beenakker, Phys. Rev. Lett. 89, 237002 (2002).

${ }^{37}$ P. G. Silvestrov, M. C. Goorden, and C. W. J. Beenakker, Phys. Rev. Lett. 90, 116801 (2003).

${ }^{38}$ M. G. Vavilov and A. I. Larkin, Phys. Rev. B 67, 115335 (2003).

${ }^{39}$ P. W. Brouwer and S. Rahav, Phys. Rev. B 74, 085313 (2006).

${ }^{40}$ T. Micklitz and A. Altland, Phys. Rev. Lett. 103, 080403 (2009).

${ }^{41} \mathrm{Ph}$. Jacquod, H. Schomerus, and C. W. J. Beenakker, Phys. Rev. Lett. 90, 207004 (2003).

${ }^{42}$ A. Kormányos, Z. Kaufmann, C. J. Lambert, and J. Cserti, Phys. Rev. B 70, 052512 (2004).

${ }^{43}$ H. Schomerus and Ph. Jacquod, J. Phys. A 38, 10663 (2005).

${ }^{44}$ M. C. Goorden, Ph. Jacquod, and C. W. J. Beenakker, Phys. Rev. B 72, 064526 (2005).

${ }^{45}$ J. Kuipers, D. Waltner, C. Petitjean, G. Berkolaiko, and K. Richter, Phys. Rev. Lett. 104, 027001 (2010).

${ }^{46}$ C. W. J. Beenakker, Phys. Rev. Lett. 67, 3836 (1991).

${ }^{47}$ G. Berkolaiko, J. M. Harrison, and M. Novaes, J. Phys. A 41, 365102 (2008).

${ }^{48}$ G. Berkolaiko and J. Kuipers, J. Phys. A 43, 035101 (2010).

${ }^{49}$ D. Waltner, J. Kuipers, and K. Richter, Phys. Rev. B 83, 195315 (2011).

${ }^{50}$ P. W. Brouwer and C. W. J. Beenakker, J. Math. Phys. 37, 4904 (1996).

${ }^{51}$ W. H. Miller, Adv. Chem. Phys. 30, 77 (1975).

${ }^{52}$ E. Akkermans, A. Auerbach, J. E. Avron, and B. Shapiro, Phys. Rev. Lett. 66, 76 (1991).

${ }^{53}$ E. Doron and U. Smilansky, Phys. Rev. Lett. 68, 1255 (1992).

${ }^{54}$ C. H. Lewenkopf and R. O. Vallejos, Phys. Rev. E 70, 036214 (2004).

${ }^{55}$ K. Richter and M. Sieber, Phys. Rev. Lett. 89, 206801 (2002).

${ }^{56}$ S. Heusler, S. Müller, P. Braun, and F. Haake, Phys. Rev. Lett. 96, 066804 (2006).

${ }^{57}$ S. Müller, S. Heusler, P. Braun, and F. Haake, New J. Phys. 9, 12 (2007).

${ }^{58}$ K. Richter, Semiclassical Theory of Mesoscopic Quantum Systems (Springer, Berlin, 2000).

${ }^{59}$ P. Braun, S. Heusler, S. Müller, and F. Haake, J. Phys. A 39, L159 (2006).

${ }^{60}$ R. S. Whitney and Ph. Jacquod, Phys. Rev. Lett. 96, 206804 (2006).

${ }^{61}$ A. Lassl, Diplomarbeit, Universität Regensburg, 2003.

${ }^{62}$ M. Sieber and K. Richter, Phys. Scr. T 90, 128 (2001).

${ }^{63}$ D. Spehner, J. Phys. A 36, 7269 (2003).

${ }^{64}$ M. Turek and K. Richter, J. Phys. A 36, L455 (2003).

${ }^{65}$ P. W. Brouwer and S. Rahav, Phys. Rev. B 74, 075322 (2006).

${ }^{66}$ W. T. Tutte, Am. Math. Mon. 71, 272 (1964).

${ }^{67}$ J. H. Hannay and A. M. Ozorio de Almeida, J. Phys. A 17, 3429 (1984).

${ }^{68}$ R. P. Stanley, Enumerative Combinatorics (Cambridge University Press, Cambridge, 2001), Vol. 2.

${ }^{69} \mathrm{P}$. W. Brouwer and C. W. J. Beenakker, Chaos Solitons Fractals 8, 1249 (1997).

${ }^{70}$ K. Saito and T. Nagao, Phys. Lett. A 352, 380 (2006).

${ }^{71}$ T. Nagao, P. Braun, S. Müller, K. Saito, S. Heusler, and F. Haake, J. Phys. A 40, 47 (2007). 
${ }^{72}$ J. Kuipers and M. Sieber, J. Phys. A 40, 935 (2007).

${ }^{73}$ R. S. Whitney and Ph. Jacquod, Phys. Rev. Lett. 94, 116801 (2005).

74 Ph. Jacquod and R. S. Whitney, Phys. Rev. B 73, 195115 (2006).

${ }^{75}$ A. Kormányos and H. Schomerus, Phys. Rev. Lett. 97, 124102 (2006).

${ }^{76}$ P. G. Silvestrov, Phys. Rev. Lett. 97, 067004 (2006).
${ }^{77}$ F. Libisch, J. Möller, S. Rotter, M. G. Vavilov, and J. Burgdörfer, Europhys. Lett. 82, 47006 (2008).

${ }^{78}$ K. M. Frahm, P. W. Brouwer, J. A. Melsen, and C. W. J. Beenakker, Phys. Rev. Lett. 76, 2981 (1996).

${ }^{79}$ S. Heusler, S. Müller, A. Altland, P. Braun, and F. Haake, Phys. Rev. Lett. 98, 044103 (2007).

${ }^{80}$ S. Müller, S. Heusler, A. Altland, P. Braun, and F. Haake, New J. Phys. 11, 103025 (2009). 